\title{
On the convergence of Dirichlet processes
}

\author{
FRANÇOIS COQUET ${ }^{1}$ and LESZEK SŁOMIŃSKI ${ }^{2}$ \\ ${ }^{1}$ Université de Rennes 1, IRMAR, Campus de Beaulieu, F-35042 Rennes, France. E-mail: \\ Francois.Coquet@univ-rennes1.fr \\ ${ }^{2}$ Faculty of Mathematics and Informatics, Nicholas Copernicus University, ul. Chopina 12/18, \\ 87-100 Toruń,Poland.E-mail: leszeks@mat.uni.torun.pl
}

For a given weakly convergent sequence $\left\{X^{n}\right\}$ of Dirichlet processes we show weak convergence of the sequence of the corresponding quadratic variation processes as well as stochastic integrals driven by the $X^{n}$ values provided that the condition UTD (a counterpart to the condition UT for Dirichlet processes) holds true. Moreover, we show that under UTD the limit process of $\left\{X^{n}\right\}$ is a Dirichlet process, too.

Keywords: Dirichlet process; stochastic integral; weak convergence

\section{Introduction}

Let $\left\{X^{n}\right\}$ be a sequence of semimartingales defined on possibly different probability spaces $\left(\Omega^{n}, F^{n}, P^{n}\right)$ and adapted to different filtrations $\mathscr{F}^{n}$, i.e. every $X^{n}$ can be decomposed into the sum of two processes:

$$
X_{t}^{n}=M_{t}^{n}+A_{t}^{n}, \quad t \in[0, T],
$$

where $M^{n}$ is an $\mathscr{F}^{n}$ local martingale and $A^{n}$ is an $\mathscr{F}^{n}$ adapted process with locally bounded variation. In the theory of convergence of semimartingales and stochastic integrals the condition UT introduced by Stricker (1985) has turned out to be very useful. In terms of conditional expectations it has the form:

UT: $\lim _{N \rightarrow+\infty} \sup _{n} P^{n}\left[\sigma^{n, N}<T\right]=0$ and for every $N \in \mathbb{N}$ the family of random variables

$$
\left\{\sum_{j=1}^{k}\left|\mathrm{E}^{n}\left(X_{s_{j}}^{n, N}-X_{s_{j-1}}^{n, N} \mid \mathscr{F}_{s_{j-1}}^{n}\right)\right| ; 0=s_{0} \leqslant \ldots \leqslant s_{k}=T, k \in \mathbb{N}, n \in \mathbb{N}\right\}
$$

is bounded in probability. Here $\sigma^{n, N}=\inf \left\{t ;\left|X_{t}^{n}\right| \geqslant N\right\}$ and $X^{n, N}$ denotes the process $X^{n}$ stopped at $\sigma^{n, N}, n \in \mathbb{N}$.

Under this condition, Jakubowski et al. (1989) proved a functional limit theorem for stochastic integrals. Next, with the use of UT, stability theorems for the stochastic differential equations were proved in the series of papers by Mémin and Słomiński (1991) 
and Słomiński (1989; 1996). On the other hand Kurtz and Protter (1991a, b) showed that employing an UT-like condition ("good sequences of semimartingales") leads to similar results on the convergence of stochastic integrals and solutions of stochastic differential equations (SDEs).

A simple example of stability theorem under UT is the following. Assume that $\left\{X^{n}\right\}$ is a sequence of continuous semimartingales and $X^{n} \rightarrow X$ in distribution in $\mathbb{C}([0, T], \mathbb{R})$. Then UT reduces to the condition

$$
\left\{\operatorname{var}\left(A^{n}\right)_{T} ; n \in \mathbb{N}\right\} \text { is bounded in probability }
$$

(where $\operatorname{var}\left(A^{n}\right)_{T}$ denotes the variation of $A^{n}$ on the interval $\left.[0, T]\right)$ and implies that $X$ is a semimartingale with respect to its natural filtration $\mathscr{F}^{X}$ and $\left(X^{n},\left[X^{n}\right]\right) \rightarrow(X,[X])$ in distribution in $\mathbb{C}\left([0, T], \mathbb{R}^{2}\right)$ (see, for example, Jacod (1980), Stricker (1985) and Jakubowski et al. (1989)).

In Section 2 we introduce a counterpart of the condition UT for sequences of continuous Dirichlet processes in the sense of Föllmer (1981) (we call it condition UTD) and we give simple characterizations of UTD. Let $\left\{D_{k}\right\}$ be a sequence of subdivisions of $[0, T]$, such that the mesh size $\left|D_{k}\right|=\max _{t_{i} \in D_{k}}\left|t_{i+1}-t_{i}\right|$ tends to 0 as $k \rightarrow+\infty$. Assume now that $\left\{X^{n}\right\}$ is a sequence of Dirichlet processes along $D_{k}$, i.e. $X^{n}$ has the decomposition of the form (1), where $M^{n}$ is an $\mathscr{F}^{n}$ local martingale but $A^{n}$ is only an $\mathscr{F}^{n}$ adapted process such that

$$
\sum_{t_{i} \in D_{k}}\left|A_{t_{i}}^{n}-A_{t_{i-1}}^{n}\right|^{2} \underset{p^{n}}{\rightarrow} 0 \quad \text { as } k \rightarrow \infty
$$

As an example we can show that, if $\left\{\sup _{t \leqslant T}\left|X_{t}^{n}\right| ; n \in \mathbb{N}\right\}$ is bounded in probability, then UTD is satisfied if and only if

$$
\left\{\sup _{t \leqslant T}\left|A_{t}^{n}\right| ; n \in \mathbb{N}\right\} \text { is bounded in probability }
$$

and

$$
\lim _{k \rightarrow \infty} \sup _{n} P^{n}\left(\sum_{t_{i} \in D_{k}}\left|A_{t_{i}}^{n}-A_{t_{i-1}}^{n}\right|^{2}>\epsilon\right)=0, \quad \epsilon>0 .
$$

Section 3 contains some stability theorems for continuous Dirichlet processes as well as for stochastic integrals driven by Dirichlet processes satisfying UTD. Suppose that $X^{n} \rightarrow X$ in distribution in $\mathbb{C}([0, T], \mathbb{R})$, where $\left\{X^{n}\right\}$ is a sequence of Dirichlet processes satisfying (2) and (3), and hence UTD. Then in Theorem 1 we show that $X$ is a Dirichlet process with respect to some filtration $\mathscr{G}$ such that $\mathscr{F}^{X} \subset \mathscr{G}$ and $\left(X^{n},\left[X^{n}\right]\right) \rightarrow(X,[X])$ in distribution in $\mathbb{C}\left([0, T], \mathbb{R}^{2}\right)$. If we want to make $X$ a Dirichlet process with respect to the given filtration $\mathscr{F}$, it is sufficient to assume that all Dirichlet processes $X^{n}$ are adapted with respect to the same filtration $\mathscr{F}$ and $X^{n} \rightarrow X$ in probability in $\mathbb{C}([0, T], \mathbb{R}$ ) (see Theorem 2).

In Section 5 we consider Dirichlet processes satisfying UTD and such that the family of random variables 


$$
\left\{\sum_{i=1}^{m}\left|A_{s_{i}}^{n}-A_{s_{i-1}}^{n}\right|^{p} ; 0=s_{0} \leqslant \ldots \leqslant s_{m}=T, s_{i} \in D_{k}, m, k, n \in \mathbb{N}\right\}
$$

is bounded in probability for some $p \in[1,2]$. In this case we say that a sequence $\left\{X^{n}\right\}$ satisfies $\operatorname{UTD}(p)$ (note that $\operatorname{UTD}(1)$ is equivalent to UT). Assume that $X, Y$ are two Dirichlet processes adapted to the same filtration and satisfying the above condition with $p<2$ and $p=2$, respectively. By using a stochastic version of some inequality of Young (1936) we prove in Theorem 3 that then it is possible to construct a stochastic integral $\int_{0} X_{s} \mathrm{~d} Y_{s}$. As a consequence we generalize slightly Bertoin's (1989) result on construction of stochastic integral for processes with bounded $p$ variation. Next, we prove a stability theorem for sequences of such stochastic integrals.

In Sections 4, 6 and 7 we give examples of sequences of processes satisfying UTD and $\mathrm{UTD}(p)$. We study functionals of Dirichlet processes, functionals of semimartingales and solutions of some special SDEs driven by continuous semimartingales. The case of diffusion processes corresponding to operators in divergence form was considered by Rozkosz and Słomiński (1998).

Now, we introduce some notation used throughout the paper. $\mathbb{C}\left([0, T], \mathbb{R}^{k}\right)$ is the space of continuous mappings $x, x: \mathbb{R}^{+} \rightarrow \mathbb{R}^{k}$, with the topology of uniform convergence on compact subsets of $\mathbb{R}^{+}$. In this paper we consider exclusively processes $X$ with trajectories in $\mathbb{C}\left([0, T], \mathbb{R}^{k}\right)$ or their discretizations, which are strictly jumping processes. Unless otherwise stated, we assume that $X(0)=0$; however, our results are also true without this restriction. Denote $D_{k}^{t}=D_{k} \cap[0, t], D_{k}^{t, s}=D_{k}^{s} \backslash D_{k}^{t}$ for all $t, s \in[0, T], t \leqslant s, k \in \mathbb{N}$. For a given process $X$ we use also the notation $\Delta X_{s_{i}}=X_{s_{i}}-X_{s_{i-1}}, s_{i} \in D_{k}, i, k \in \mathbb{N}$, and [X] denotes a quadratic variation process of $X$ along $D_{k}$, i.e. for $t \in[0, T],[X]_{t}$ is a limit in probability of the sequence $\left\{\sum_{t_{i} \in D_{k}^{t}}\left|\Delta X_{t_{i}}^{n}\right|^{2}\right\}_{k \in N}$. Finally, $\rightarrow \mathscr{D}$ and $\rightarrow_{P}$ denote convergence in law and in probability, respectively.

\section{Dirichlet processes and condition UTD}

Definition 1. We call $X$ an $\mathscr{F}$ Dirichlet process if it admits the decomposition

$$
X_{t}=M_{t}+A_{t}, \quad t \in[0, T],
$$

where $M$ is an $\mathscr{F}$ local martingale and $A$ is an $\mathscr{F}$ adapted process of 0 -quadratic variation along $D_{k}$, i.e.

$$
\sum_{t_{i} \in D_{k}}\left|\Delta A_{t_{i}}\right|^{2} \underset{P}{\rightarrow} 0, \quad \text { as } k \rightarrow+\infty
$$

Note that, in the original definition of Föllmer (1981), $M$ and $A$ in the decomposition (5) are square integrable and (6) has the form $\mathrm{E} \sum_{t_{i} \in D_{k}}\left|\Delta A_{t_{i}}\right|^{2} \rightarrow 0$.

In the paper we also consider processes with bounded $p$ variation in the following sense.

Definition 2. We say that an $\mathscr{T}$ Dirichlet process $X=M+A$ is of class $\mathscr{D}^{p}$ for $p \in[1,2]$ if 
additionally the family of random variables

$$
\left\{\sum_{i=1}^{m}\left|\Delta A_{s_{i}}\right|^{p} ; 0=s_{0} \leqslant \ldots \leqslant s_{m}=T, s_{i} \in D_{k}, m, k \in \mathbb{N}\right\}
$$

is bounded in probability.

Note that Bertoin $(1986,1989)$ developed a theory of Dirichlet processes assuming instead of (6) and (7) slightly more restrictive assumptions

$$
\lim _{|\mathscr{T}| \rightarrow 0} \mathrm{E} \sum_{i=1}^{+\infty}\left|X_{\sigma_{i}}-X_{\sigma_{i-1}}\right|^{2}=0
$$

for all subdivisions $[0, T]$ by sequences of $\mathscr{F}_{t}$ stopping times $\mathscr{T}=\left(0=\sigma_{0} \leqslant \sigma_{1} \leqslant\right.$ $\ldots \leqslant T)$ such that $|\mathscr{T}|=\operatorname{Esup}_{i}\left(\sigma_{i}-\sigma_{i-1}\right) \rightarrow 0$ and

$$
\sup _{\mathscr{T}} \mathrm{E}\left(\sum_{i=1}^{+\infty}\left|X_{\sigma_{i}}-X_{\sigma_{i-1}}\right|^{p}\right)^{1 / p}<+\infty
$$

respectively.

It is clear that the families of Dirichlet processes $\mathscr{D}^{p}$ introduced above have the following simple properties: $\mathscr{D}^{1}$ is a family of $\mathscr{F}$ semimartingales and for $1 \leqslant p \leqslant p^{\prime} \leqslant 2$ we have $\mathscr{D}^{p} \subset \mathscr{D}^{p^{\prime}} \subset \mathscr{D}^{2}$. It is also straightforward to observe that $\mathscr{D}^{2}$ consists of Dirichlet processes $X$ such that the family of random variables

$$
\left\{\sum_{i=1}^{m}\left|\Delta X_{s_{i}}\right|^{2} ; 0=s_{0} \leqslant \ldots \leqslant s_{m}=T, s_{i} \in D_{k}, m, k \in \mathbb{N}\right\}
$$

is bounded in probability. Note that the class of Dirichlet processes is larger than $\mathscr{D}^{2}$, as the following deterministic example shows.

Example 1. Take the sequence of dyadic subdivisions of $[0,1]: D_{k}=\left\{j 2^{-k}, 0 \leqslant j \leqslant 2^{k}\right\}$ and let $X$ be the process defined for all integer $p \geqslant 1$ by

$$
X_{t}= \begin{cases}\frac{2^{2 p}}{p^{1 / 2}}\left(t-1+2^{1-2 p}\right) & \text { if } t \in\left[1-2^{1-2 p}, 1-2^{-2 p}\right], \\ \frac{-2^{2 p+1}}{p^{1 / 2}}\left(t-1+2^{-2 p-1}\right) & \text { if } t \in\left[1-2^{-2 p}, 1-2^{-2 p-1}\right], \\ 0 & \text { otherwise. }\end{cases}
$$

Then, $X$ is a piecewise affine function, equal to 0 at each $t=1-2^{1-2 p}$ and such that $X_{t}=1 / p^{1 / 2}$ for $t=1-2^{-2 p}$. If $S_{N}=\left\{1-2^{-2 k}, k \leqslant 2 N\right\}$, then $S_{N} \subset D_{2 N+1}$ and

$$
\sum_{s_{j} \in S_{N}}\left|\Delta X_{s_{j}}\right|^{2}=2 \sum_{1 \leqslant i \leqslant N} \frac{1}{i} \text {. }
$$


This sum is of order in $N$, which is unbounded; hence $X$ does not belong to $\mathscr{D}^{2}$. On the other hand, we can explicitly compute the 2-variation of $X$ along $D_{k}$ :

$$
\begin{aligned}
\sum_{t_{i} \in D_{k}}\left|\Delta X_{t_{i}}\right|^{2} & =\sum_{p=1}^{[k / 2]}\left[\sum_{t_{i} \in\left[1-2^{-2} p+1,1-2^{-2 p}\right] \cap D_{k}}\left|\Delta X_{t_{i}}\right|^{2}+\sum_{t_{i} \in\left[1-2^{-2}, 1-2^{-2 p-1}\right] \cap D_{k}}\left|\Delta X_{t_{i}}\right|^{2}\right] \\
& =\sum_{p=1}^{[k / 2]}\left[2^{k-2 p}\left[\frac{2^{2 p-k}}{p^{1 / 2}}\right)^{2}+2^{k-2 p-1}\left(\frac{2^{2 p+1-k}}{p^{1 / 2}}\right)^{2}\right] \\
& =3 \times 2^{-k} \sum_{p=1}^{[k / 2]} \frac{2^{2 p}}{p} .
\end{aligned}
$$

Now, for fixed $\epsilon>0$ and every integer $l, 4 \epsilon \leqslant l<k / 2$,

$$
2^{-k} \sum_{p=1}^{[k / 2]} \frac{2^{2 p}}{p} \leqslant \frac{1}{l+1} \sum_{p=l+1}^{[k / 2]} 2^{2 p-k}+2^{-k} \sum_{p=1}^{l} \frac{2^{2 p}}{p} \leqslant \frac{\epsilon}{2}+2^{-k} \times \text { constant }
$$

and, for $k$ sufficiently large,

$$
2^{-k} \sum_{p=1}^{[k / 2]} \frac{2^{2 p}}{p} \leqslant \epsilon
$$

i.e. $X$ is a Dirichlet process.

Let $\left\{X^{n}\right\}$ be a sequence of continous processes defined possibly on different probability spaces $\left(\Omega^{n}, F^{n}, P^{n}\right)$ and adapted to different filtrations $\mathscr{F}^{n}, n \in \mathbb{N}$. We say that the sequence $\left\{X^{n}\right\}$ satisfies the condition UTD if

UTD: (i) $\lim _{N \rightarrow+\infty} \sup _{n} P^{n}\left(\sigma^{n, N}<T\right)=0$ and

$$
\lim _{k \rightarrow+\infty} \sup _{n} \sup _{j \geqslant k} P^{n}\left(\sum_{t_{i} \in D_{k}}\left|\sum_{u_{l} \in D_{j}^{t_{i-1}, t_{i}}} \mathrm{E}^{n}\left(\Delta X_{u_{l}}^{n, N} \mid \mathscr{F}_{u_{l-1}}^{n}\right)\right|^{2}>\epsilon\right)=0, \quad \epsilon>0, N \in \mathbb{N},
$$

where $\sigma^{n, N}=\inf \left\{t ;\left|X_{t}^{n}\right| \geqslant N\right\}$ and $X^{n, N}$ denotes the process $\mathrm{X}^{n}$ stopped at $\sigma^{n, N}$, and $D_{k} \subset D_{k+1}, k \in N$,

(ii) $\left\{\sum_{t_{i} \in D_{k}}\left|\Delta X_{t_{i}}^{n}\right|^{2} ; k, n \in \mathbb{N}\right\}$ is bounded in probability.

Similarly we say that a sequence of continous processes $\left\{X^{n}\right\}$ satisfies UTD(2) if $\mathrm{UTD}(2)$ : the condition (i) of UTD holds and the family of random variables

$$
\left\{\sum_{i=1}^{m}\left|\Delta X_{s_{i}}^{n}\right|^{2} ; 0=s_{0} \leqslant \ldots \leqslant s_{m}=T, s_{i} \in D_{k}, m, k, n \in \mathbb{N}\right\}
$$

is bounded in probability. 
Remark 1. By using the arguments from Föllmer (1981) one can prove that if the condition UTD is satisfied then for every $n \in \mathbb{N}, X^{n}$ is an $\mathscr{F}^{n}$ Dirichlet process in the sense of Definition 1, i.e.

$$
X_{t}^{n}=M_{t}^{n}+A_{t}^{n}, \quad t \in[0, T],
$$

where $M^{n}$ is an $\mathscr{F}^{n}$ local martingale and $A^{n}$ is an $\mathscr{F}^{n}$ adapted process of 0 -quadratic variation along $D_{k}$.

Proposition 1. Let $\left\{X^{n}\right\}$ be a sequence of $\mathscr{F}_{t}^{n}$ adapted processes. The following two conditions are equivalent.

(i) $\left\{X^{n}\right\}$ satisfies UTD (on UTD(2)).

(ii) $\left\{X^{n}=M^{n}+A^{n}\right\}$ is a sequence of Dirichlet processes such that (3) holds true and the families of random variables $\left\{\left[M^{n}\right]_{T}\right\},\left\{\sup _{t \leqslant T}\left|A_{t}^{n}\right|\right\}$ are bounded in probability (on (3) and (4) with $p=2$ hold true and $\left\{\left[M^{n}\right]_{T}\right\}$ is bounded in probability).

Proof. (ii) $\Rightarrow$ (i) By the boundedness in probability of $\left\{\left[M^{n}\right\}_{T}\right\},\left\{\sup _{t \leqslant T}\left|A_{t}^{n}\right|\right\}$ we deduce that the sequence $\left\{\sup _{t \leqslant T}\left|X_{t}^{n}\right|\right\}$ is bounded in probability as well, and in particular,

$$
\lim _{N \rightarrow+\infty} \sup _{n} P^{n}\left(\sigma^{n, N^{*}}<T\right)=0
$$

where $\sigma^{n, N^{*}}=\inf \left\{t ;\left|A_{t}^{n}\right|>N\right\} \wedge \sigma^{n, N}, n, N \in \mathbb{N}$. In the sequel for every $n, N \in \mathbb{N}$ we write $X^{n, N^{*}}, M^{n, N^{*}}, A^{n, N^{*}}$ to denote the processes $X^{n}, M^{n}, A^{n}$ stopped at $\sigma^{n, N^{*}}$.

By Itô's formula,

$$
\sum_{t_{i} \in D_{k}}\left|\Delta M_{t_{i}}^{n, N^{*}}\right|^{2}=2 \sum_{t_{i} \in D_{k}} \int_{\left(t_{i-1}, t_{i}\right]}\left(M_{s}^{n, N^{*}}-M_{t_{i-1}}^{n, N^{*}}\right) \mathrm{d} M_{s}^{n, N^{*}}+\left[M^{n, N^{*}}\right]_{T}, \quad n \in \mathbb{N},
$$

and for every $C, N$, by the Chebyshev inequality, $P^{n}\left(\sum_{t_{i} \in D_{k}}\left|\Delta M_{t_{i}}^{n, N^{*}}\right|^{2}>C\right) \leqslant 4 N^{2} / C^{2}$. Therefore

$$
P^{n}\left(\sum_{t_{i} \in D_{k}}\left|\Delta X_{t_{i}}^{n}\right|^{2}>2 C\right) \leqslant \frac{4 N^{2}}{C^{2}}+P^{n}\left(\sigma^{n, N,{ }^{*}}<T\right)+P^{n}\left(\sum_{t_{i} \in D_{k}}\left|\Delta A_{t_{i}}^{n}\right|^{2}>C\right)
$$

hence the condition (ii) of UTD follows by (3) and (10).

If we denote $\gamma_{k}^{n, N^{*}}=\inf \left\{t ; \sum_{t_{i} \in D_{k}^{t}}\left|\Delta A_{t_{i}}^{n, N^{*}}\right|^{2}>1\right\}$ for $n, k, N \in \mathbb{N}$, then using once more (3) and (10) it is clear that

$$
\lim _{k \rightarrow \infty} \sup _{n} P^{n}\left(\gamma_{k}^{n, N^{*}}<T\right)=0, \quad N \in \mathbb{N}
$$

and that

$$
\lim _{k \rightarrow+\infty} \sup _{n} \mathrm{E}^{n} \sum_{t_{i} \leqslant \gamma_{k}^{n, N^{*}}}\left|\Delta A_{t_{i}}^{n, N^{*}}\right|^{2}=0, \quad N \in \mathbb{N} .
$$

On the other hand, by standard arguments, for $j \geqslant k$, 


$$
\begin{aligned}
& \mathrm{E}^{n} \sum_{t_{i} \in D_{K}}\left|\sum_{u_{i} \in D_{j}^{t_{i-1}, t_{i}}, u_{l} \leqslant \gamma_{j}^{n, N^{*}}} \mathrm{E}^{n}\left(\Delta X_{u_{l}}^{n, N^{*}} \mid \mathscr{F}_{u_{l-1}}^{n}\right)\right|^{2} \\
& \quad \leqslant 2 \mathrm{E}^{n}\left(\sum_{t_{i} \in D_{K}}\left|\sum_{u_{i} \in D_{j}^{t_{i-1}, t_{i}}, u_{l} \leqslant \gamma_{j}^{n, N^{*}}} \mathrm{E}^{n}\left(\Delta X_{u_{l}}^{n, N^{*}} \mid \mathscr{F}_{u_{l-1}}^{n}\right)-\Delta A_{u_{l}}^{n, N}\right|^{2}\right)+2 \mathrm{E}^{n} \sum_{t_{i} \leqslant \gamma_{k}^{n, N^{*}}}\left|\Delta A_{t_{i}}^{n, N^{*}}\right|^{2} \\
& \quad \leqslant 2 \mathrm{E}^{n}\left(\sum_{u_{i} \in D_{j}^{t_{i-1}, t_{i}}, u_{l} \leqslant \gamma_{j}^{n, N^{*}}}\left|\mathrm{E}^{n}\left(\Delta A_{u_{l}}^{n, N^{*}} \mid \mathscr{F}_{u_{l-1}}^{n}\right)-\Delta A_{u_{l}}^{n, N^{*}}\right|^{2}\right)+2 \mathrm{E}^{n} \sum_{t_{i} \leqslant \gamma_{k}^{n, N^{*}}}\left|\Delta A_{t_{i}}^{n, N^{*}}\right|^{2} \\
& \quad \leqslant 2 \mathrm{E}^{n}\left(\sum_{u_{i} \in D_{j}^{t_{i-1}, t_{i}}, u_{l} \leqslant \gamma_{j}^{n, N^{*}}}\left|\Delta A_{u_{l}}^{n, N^{*}}\right|^{2}\right)+2 \mathrm{E}^{n} \sum_{t_{i} \leqslant \gamma_{k}^{n, N^{*}}}\left|\Delta A_{t_{i}}^{n, N^{*}}\right|^{2} .
\end{aligned}
$$

Therefore, by (12),

$$
\sup _{n} \sup _{j \geqslant k} \mathrm{E}^{n} \sum_{t_{i} \in D_{K}}\left|\sum_{u_{i} \in D_{j}^{t_{i-1}, t_{i}}, u_{l} \leqslant \gamma_{j}^{n, N^{*}}} \mathrm{E}^{n}\left(\Delta X_{u_{l}}^{n, N^{*}} \mid \mathscr{F}_{u_{l-1}}^{n}\right)\right|^{2} \rightarrow 0, \quad \text { as } k \rightarrow+\infty
$$

which gives the condition (i) of UTD, when combined with (10) and (11).

Assume additionally that the condition (4) is satisfied with $p=2$. Since for every subdivision $0=s_{0} \leqslant s_{1} \leqslant \ldots \leqslant s_{m}=T$ such that $s_{i} \in D_{k}, m, k \in \mathbb{N}$

$$
P^{n}\left(\sum_{i=1}^{m}\left|\Delta X_{s_{i}}^{n}\right|^{2}>2 C\right) \leqslant \frac{4 N^{2}}{C^{2}}+P^{n}\left(\sigma^{n, N^{*}}<T\right)+P^{n}\left(\sum_{i=1}^{m}\left|\Delta A_{s_{i}}^{n}\right|^{2}>C\right),
$$

it follows by (10) that the condition $\operatorname{UTD}(2)$ holds true.

(i) $\Rightarrow$ (ii) by the method previously used by Föllmer (1981) one can show that, for every $n \in \mathbb{N}, X^{n}$ is an $\mathscr{F}^{n}$ Dirichlet process, i.e. $X_{t}^{n}=M_{t}^{n}+A_{t}^{n}$, where $M^{n}$ is an $\mathscr{F}^{n}$ local martingale and $A^{n}$ is an $\mathscr{F}^{n}$ adapted process of 0 -quadratic variation along $D_{k}$ such that

$$
\lim _{k \rightarrow+\infty} \sup _{n} P^{n}\left(\sup _{t \leqslant T}\left|\sum_{t_{i} \in D_{k}^{t}} \mathrm{E}^{n}\left(\Delta X_{t_{i}}^{n, N} \mid \mathscr{F}_{t_{i-1}}^{n}\right)-A_{t}^{n, N}\right|>\epsilon\right)=0, \quad \epsilon>0, N \in \mathbb{N},
$$

which implies that

$$
\lim _{k \rightarrow+\infty} \sup _{n} P^{n}\left(\sup _{t \leqslant T} \sum_{t_{i} \in D_{k}^{t}}\left|\mathrm{E}^{n}\left(\Delta X_{t_{i}}^{n, N} \mid \mathscr{F}_{t_{i-1}}^{n}\right)-\Delta A_{t_{i}}^{n, N}\right|^{2}>\epsilon\right)=0, \quad \epsilon>0, N \in \mathbb{N}
$$

Therefore 


$$
\begin{gathered}
\sup _{n} P^{n}\left(\sum_{t_{i} \in D_{k}}\left|\Delta A_{t_{i}}^{n, N}\right|^{2}>\epsilon\right) \leqslant \sup _{n} P^{n}\left(\sum_{t_{i} \in D_{k}} 2\left|\mathrm{E}^{n}\left(\Delta X_{t_{i}}^{n, N} \mid \mathscr{F}_{t_{i-1}}^{n}\right)-\Delta A_{t_{i}}^{n, N}\right|^{2}>\frac{\epsilon}{2}\right) \\
\quad+\sup _{n} P^{n}\left(\sum_{t_{i} \in D_{k}} 2\left|\mathrm{E}^{n}\left(\Delta X_{t_{i}}^{n, N} \mid \mathscr{F}_{t_{i-1}}^{n}\right)\right|^{2}>\frac{\epsilon}{2}\right) ;
\end{gathered}
$$

hence we conclude from (13) that

$$
\lim _{k \rightarrow+\infty} \sup _{n} P^{n}\left(\sum_{t_{i} \in D_{k}}\left|\Delta A_{t_{i}}^{n, N}\right|^{2}>\epsilon\right) \leqslant \lim _{k \rightarrow+\infty} \sup _{n} P^{n}\left(\sum_{t_{i} \in D_{k}}\left|\mathrm{E}^{n}\left(\Delta X_{t_{i}}^{n, N} \mid \mathscr{F}_{t_{i-1}}^{n}\right)\right|^{2}>\frac{\epsilon}{2}\right) .
$$

Applying (i) of UTD yields (3). Similarly, for every $C \in \mathbb{R}^{+}$,

$$
P^{n}\left(\sup _{t \leqslant T}\left|A_{t}^{n, N}\right|^{2}>C\right) \leqslant \liminf _{k \rightarrow+\infty} P^{n}\left(\sup _{t \leqslant T} 2\left|\sum_{t_{i} \in D_{k}^{t}} \mathrm{E}^{n}\left(\Delta X_{t_{i}}^{n, N} \mid \mathscr{F}_{t_{i-1}}^{n}\right)\right|^{2}>\frac{C}{2}\right) .
$$

Let $\delta_{k, C}^{n, N}=\inf \left\{t ; \sum_{t_{i} \in D_{k}^{t}}\left|\Delta X_{t_{i}}^{n, N}\right|^{2}>C^{1 / 2}\right\}$ for $n, k, N \in \mathbb{N}$ and $C \in \mathbb{R}^{+}$. By applying Doob's inequality to the discrete martingale $\sum_{t_{i} \in D_{k}^{t}}\left\{\mathrm{E}^{n}\left(\Delta X_{t_{i}}^{n, N} \mid \mathscr{F}_{t_{i-1}}^{n}\right)-\Delta X_{t_{i}}^{n, N}\right\}$ we have

$$
\begin{aligned}
& P^{n}\left(\sup _{t \leqslant T} 2\left|\sum_{t_{i} \in D_{k}^{t}} \mathrm{E}^{n}\left(\Delta X_{t_{i}}^{n, N} \mid \mathscr{F}_{t_{i-1}}^{n}\right)\right|^{2}>\frac{C}{2}\right) \\
& \quad \leqslant P^{n}\left(\sup _{t \leqslant T} 4\left|\sum_{t_{i} \in D_{k}^{t}}\left\{\mathrm{E}^{n}\left(\Delta X_{t_{i}}^{n, N} \mid \mathscr{F}_{t_{i-1}}^{n}\right)-\Delta X_{t_{i}}^{n, N}\right\}\right|^{2}>\frac{C}{4}\right)+P^{n}\left(\sup _{t \leqslant T} 4\left|X_{t}^{n, N}\right|^{2}>\frac{C}{4}\right) \\
& \leqslant P^{n}\left(\delta_{k, C}^{n, N}<T\right)+\frac{4}{C} \mathrm{E}^{n} \sum_{t_{i} \leqslant \delta_{k, C}^{n, N}}\left|\Delta X_{t_{i}}^{n, N}\right|^{2}+P^{n}\left(\sup _{t \leqslant T} 4\left|X_{t}^{n, N}\right|^{2}>\frac{C}{4}\right) \\
& \leqslant P^{n}\left(\delta_{k, C}^{n, N}<T\right)+\frac{4}{C}\left(C^{1 / 2}+4 N^{2}\right),
\end{aligned}
$$

provided that $C \geqslant 16 N^{2}$. Since owing to UTD (ii)

$$
\lim _{C \rightarrow+\infty} \sup _{k} \sup _{n} P^{n}\left(\delta_{k, C}^{n, N}<T\right)=0, \quad N \in \mathbb{N}
$$

it follows by (12) that $\lim _{C \rightarrow+\infty} \sup _{n} P^{n}\left(\sup _{t \leqslant T}\left|A_{t}^{n, N}\right|^{2}>C\right)=0, N \in \mathbb{N}$. Consequently, the families of random variables $\left\{\sup _{t \leqslant T}\left|A_{t}^{n}\right|\right\}$ as well as $\left\{\sup _{t \leqslant T}\left|M_{t}^{n}\right|\right\}$ are bounded in probability (by UTD (i) $\lim _{C \rightarrow+\infty} \sup _{n} P^{n}\left(\sup _{t \leqslant T}\left|X_{t}^{n}\right|>C\right.$ ) $=0$ ). Hence we deduce also boundedness in probability of $\left\{\left[M^{n}\right]_{T}\right\}$ and the first assertion is proved.

Now, assume additionally that the family of random variables

$$
\left\{\sum_{i=1}^{m}\left|\Delta X_{s_{i}}^{n}\right|^{2} ; 0=s_{0} \leqslant \ldots \leqslant s_{m}=T, s_{i} \in D_{k}, m, k, n \in \mathbb{N}\right\}
$$

is bounded in probability. Since boundedness in probability of 


$$
\left\{\sum_{i=1}^{m}\left|\Delta M_{s_{i}}^{n}\right|^{2} ; 0=s_{0} \leqslant \ldots \leqslant s_{m}=T, s_{i}, \in S_{k}, m, k, n \in \mathbb{N}\right\}
$$

is implied by the boundedness of $\left\{\sup _{t \leqslant T}\left|M_{t}^{n}\right|\right\}$, the condition (4) with $p=2$ holds true and the proof is complete.

It is possible to give a slightly simpler characterization of UTD in the case when the sequence $\left\{X^{n}\right\}$ is such that $\left\{\sup _{t \leqslant T}\left|X_{t}^{n}\right| ; n \in \mathbb{N}\right\}$ is bounded in probability.

Corollary 1. Assume that $\left\{X^{n}\right\}$ is a sequence of processes such that $\left\{\sup _{t \leqslant T}\left|X_{t}^{n}\right| ; n \in \mathbb{N}\right\}$ is bounded in probability. $\left\{X^{n}\right\}$ satisfies UTD (or UTD(2)) if and only if (2) and (3) hold true (or (4) with $p=2$ and (3) hold true).

Proof. From the boundedness in probability of $\left\{\sup _{t \leqslant T}\left|X_{t}^{n}\right|\right\}$ and $\left\{\sup _{t \leqslant T}\left|A_{t}^{n}\right|\right\}$ we deduce the same property also for $\left\{\left[M^{n}\right]_{T}\right\}$. Owing to Proposition 1 the proof is complete.

\section{Stability of Dirichlet processes}

In this section the main stability theorems for a sequence of Dirichlet processes $\left\{X^{n}\right\}$ are given. First, we consider the case when the sequence $\left\{X^{n}\right\}$ is weakly convergent.

Theorem 1. Assume that $\left\{X^{n}\right\}$ is a sequence of Dirichlet processes satisfying UTD (or $\mathrm{UTD}(2))$. If $X^{n} \rightarrow_{\mathscr{D}} X$ in $\mathbb{C}([0, T], \mathbb{R})$ then and

(i) $X$ is a $\mathscr{G}$ Dirichlet process for some filtration $\mathscr{G}$ such that $\mathscr{F}^{X} \subset \mathscr{G}\left(\right.$ or $\left.X \in \mathscr{D}^{2}\right)$

$$
\left(X^{n},\left[X^{n}\right]\right) \underset{\mathscr{D}}{\rightarrow}(X,[X]) \quad \text { in } \mathbb{C}\left([0, T], \mathbb{R}^{2}\right),
$$

(ii) if $f \in \mathscr{C}^{1}$, then

$$
\left(X^{n}, \int_{0} f\left(X_{s}^{n}\right) \mathrm{d} X_{s}^{n}\right) \underset{\mathscr{D}}{\longrightarrow}\left(X, \int_{0} f\left(X_{s}\right) \mathrm{d} X_{s}\right) \quad \text { in } \mathbb{C}\left([0, T], \mathbb{R}^{2}\right),
$$

(iii) if $\left\{Y^{n}\right\}$ is another sequence of Dirichlet processes satisfying UTD with respect to the same filtrations as $\left\{X^{n}\right\}$ and $\left(X^{n}, Y^{n}\right) \rightarrow_{\mathscr{D}}(X, Y)$ in $\mathbb{C}\left([0, T], \mathbb{R}^{2}\right)$, then

$$
\left(X^{n}, Y^{n},\left[X^{n}\right],\left[Y^{n}\right],\left[X^{n}, Y^{n}\right]\right) \underset{\mathscr{D}}{\rightarrow}(X, Y,[X],[Y],[X, Y]) \quad \text { in } \mathbb{C}\left([0, T], \mathbb{R}^{5}\right)
$$

\section{Proof.}

(i) Let us start by proving that

$$
\sup _{n} P^{n}\left(\left.\sup _{t \leqslant T}\left|\sum_{t_{i} \in D_{k}^{t}}\right| \Delta X_{t_{i}}^{n}\right|^{2}-\left[X^{n}\right]_{t} \mid>\epsilon\right) \rightarrow 0, \quad \text { as } k \rightarrow+\infty .
$$


It is evident that

$$
\sum_{t_{i} \in D_{k}^{t}}\left|\Delta X_{t_{i}}^{n}\right|^{2}=\sum_{t_{i} \in D_{k}^{t}}\left|\Delta M_{t_{i}}^{n}\right|+2 \sum_{t_{i} \in D_{k}^{t}} \Delta M_{t_{i}}^{n} \Delta A_{t_{i}}^{n}+\sum_{t_{i} \in D_{k}^{t}}\left|\Delta A_{t_{i}}^{n}\right|^{2} .
$$

Owing to Proposition 1,

$$
\sup _{n} P^{n}\left(\left.\sup _{t \leqslant T}\left|\sum_{t_{i} \in D_{k}^{t}}\right| \Delta X_{t_{i}}^{n}\right|^{2}-\sum_{t_{i} \in D_{k}^{t}}\left|\Delta M_{t_{i}}^{n}\right|^{2} \mid>\epsilon\right) \rightarrow 0, \quad \text { as } k \rightarrow+\infty .
$$

On the other hand by Itô's formula for $t \in D_{k}$,

$$
\left[X^{n}\right]_{t}=\left[M^{n}\right]_{t}=\sum_{t_{i} \in D_{k}^{t}}\left|\Delta M_{t_{i}}^{n}\right|^{2}-2 \sum_{t_{i} \in D_{k}^{t}} \int_{\left(t_{i-1}, t_{i}\right]}\left(M_{s}^{n}-M_{t_{i-1}}^{n}\right) \mathrm{d} M_{s}^{n} .
$$

By tightness of $\left\{X^{n}\right\}$ and by (3), $\sup _{n} P^{n}\left(\max _{t_{i} \in D_{k}}\left|\Delta M_{t_{i}}^{n}\right|>\epsilon\right) \rightarrow 0$, as $k \rightarrow+\infty$. Denote $N_{t_{i}}^{n}=\sup _{s \in\left(t_{i-1}, t_{i}\right]}\left|M_{s}^{n}-M_{t_{i-1}}^{n}\right|, n \in \mathbb{N}, t_{i} \in D_{k}$. We shall also show that

$$
\sup _{n} P^{n}\left(\max _{t_{i} \in D_{k}}\left|N_{t_{i}}^{n}\right|>\epsilon\right) \rightarrow 0, \quad \text { as } k \rightarrow+\infty .
$$

Assume for simplicity that $\sup _{t \leqslant T}\left|M_{t}^{n}\right| \leqslant C, n \in \mathbb{N}$. By the lemma of Dvoretzky for every $\epsilon, \delta>0$

$$
P^{n}\left(\max _{t_{i} \in D_{k}}\left|N_{t_{i}}^{n}\right|>\epsilon\right) \leqslant \delta+P^{n}\left(\sum_{t_{i} \in D_{k}} P^{n}\left(N_{t_{i}}^{n}>\epsilon \mid \mathscr{F}_{t_{i-1}}^{n}\right)>\delta\right) .
$$

Since, owing to the Chebyshev inequality for continuous martingales, for every $\epsilon, \eta>0$,

$$
\begin{aligned}
\sum_{t_{i} \in D_{k}} P^{n}\left(N_{t_{i}}^{n}>\epsilon \mid \mathscr{F}_{t_{i-1}}^{n}\right) \leqslant & \epsilon^{-4} \sum_{t_{i} \in D_{k}^{t}} \mathrm{E}^{n}\left(\left|M_{t_{i}}^{n}-M_{t_{i-1}}^{n}\right|^{4} \mid \mathscr{F}_{t_{i-1}}^{n}\right) \\
\leqslant & \eta^{2} \epsilon^{-4} \sum_{t_{i} \in D_{k}^{t}} \mathrm{E}^{n}\left(\left|M_{t_{i}}^{n}-M_{t_{i-1}}^{n}\right|^{2} \mid \mathscr{F}_{t_{i-1}}^{n}\right) \\
& +(2 C)^{4} \epsilon^{-4} \sum_{t_{i} \in D_{k}} P^{n}\left(\left|M_{t_{i}}^{n}-M_{t_{i-1}}^{n}\right|>\eta \mid \mathscr{F}_{t_{i-1}}^{n}\right)
\end{aligned}
$$

and $\quad \mathrm{E}^{n} \sum_{t_{i} \in D_{k}} P^{n}\left(\left|M_{t_{i}}^{n}-M_{t_{i-1}}^{n}\right|>\eta \mid \mathscr{F}_{t_{i-1}}^{n}\right)=P^{n}\left(\max _{t_{i} \in D_{k}}\left|M_{t_{i}}^{n}-M_{t_{i-1}}^{n}\right|>\eta\right)$, the property (16) follows. Therefore

$$
\lim _{k \rightarrow+\infty} \sup _{n} P^{n}\left(\sup _{t \leqslant T} \sum_{t_{i} \in D_{k}^{t}}\left|\int_{\left(t_{i-1}, t_{i}\right]}\left(M_{s}^{n}-M_{t_{i-1}}^{n}\right) \mathrm{d} M_{s}^{n}\right|>\epsilon\right)=0, \quad \epsilon>0,
$$

and the proof of (14) is completed.

On the other hand note that, for every $k \in \mathbb{N},\left\{\sum_{t_{i} \in D_{k}^{t}}\left|\Delta X_{t_{i}}^{n}\right|^{2}\right\}$ is a sequence of strictly jumping processes with cadlag trajectories. Since $X^{n} \rightarrow_{\mathscr{D}} X$ in $\mathbb{C}([0, T], \mathbb{R})$, 


$$
\sum_{t_{i} \in D_{k}}\left|\Delta X_{t_{i}}^{n}\right|^{2} \underset{\mathscr{D}}{\longrightarrow} \sum_{t_{i} \in D_{k}}\left|\Delta X_{t_{i}}\right|^{2}, \quad k \in \mathbb{N}
$$

in the Skorokhod topology $\mathrm{J}_{1}$ (see, for example, Jacod and Shiryaev (1987, Chapter VI)). The task is now to observe that $X$ is a process possessing the quadratic variation process $[X]$. Let $j \geqslant k$. Then, by (17) and (14),

$$
\begin{aligned}
& P\left(\left.\sup _{t \leqslant T}\left|\sum_{t_{i} \in D_{k}^{t}}\right| \Delta X_{t_{i}}\right|^{2}-\sum_{t_{i} \in D_{k}^{t}} \sum_{u_{l} \in D_{j}^{t_{i-1}, t_{i}}}\left|\Delta X_{u_{l}}\right|^{2} \mid>\epsilon\right) \\
& \quad \leqslant \liminf _{n \rightarrow+\infty} P^{n}\left(\left.\sup _{t \leqslant T}\left|\sum_{t_{i} \in D_{k}^{t}}\right| \Delta X_{t_{i}}^{n}\right|^{2}-\sum_{t_{i} \in D_{k}^{t}} \sum_{u_{l} \in D_{j}^{t_{i-1}, t_{i}}}\left|\Delta X_{u_{l}}^{n}\right|^{2} \mid>\epsilon\right) \\
& \quad \leqslant \sup _{n} 2 P^{n}\left(\left.\sup _{t \leqslant T}\left|\left[X^{n}\right]_{t}-\sum_{t_{i} \in D_{k}^{t}} \sum_{u_{l} \in D_{j}^{t_{i-1}, t_{i}}}\right| \Delta X_{u_{l}}^{n}\right|^{2} \mid>\epsilon\right) \\
& \rightarrow 0, \quad \text { as } k \rightarrow \infty .
\end{aligned}
$$

Hence $\left\{\sum_{t_{i} \in D_{k}^{t}}\left|\Delta X_{t_{i}}\right|^{2}\right\}_{k \in \mathbb{N}}$ is a Cauchy sequence for the distance in probability and there exists a process $[X]_{t}$ such that $\left.\sup _{t \leqslant T}\left|\sum_{t_{i} \in D_{k}^{t}}\right| \Delta X_{t_{i}}\right|^{2}-[X]_{t} \mid \rightarrow_{P} 0$. Using once more (17) and (14) we obtain the convergence

$$
\left(X^{n},\left[X^{n}\right]\right) \underset{\mathscr{D}}{\rightarrow}(X,[X]) \quad \text { in } \mathbb{C}\left([0, T], \mathbb{R}^{2}\right) .
$$

Since $[X]$ is the process with continuous trajectories and $\left[M^{n}\right]=\left[X^{n}\right]$, for every sequence $\left\{\tau_{n}\right\}$ of stopping times, $\tau_{n} \leqslant T$ and, for every sequence of constants $\left\{\delta_{n}\right\}$ such that $\delta_{n} \downarrow 0$,

$$
\left[M^{n}\right]_{\left(\tau_{n}+\delta_{n}\right) \wedge T}-\left[M^{n}\right]_{\tau_{n}} \underset{P}{\longrightarrow} 0 .
$$

In view of the Aldous (1978) criterion the sequence $\left\{\left[M^{n}\right]\right\}$ is tight in $\mathbb{C}([0, T], \mathbb{R})$, which implies that

$$
\left\{\left(X^{n}, M^{n}\right)\right\} \text { is tight in } \mathbb{C}\left([0, T], \mathbb{R}^{2}\right) .
$$

Assume that along some subsequence $\left(n^{\prime}\right) \subset(n)\left(X^{n^{\prime}}, M^{n^{\prime}}\right) \rightarrow_{\mathscr{D}}(X, M)$. Then it is easily seen that $M$ is a local martingale with respect to $\mathscr{G}=\mathscr{F}^{(X, M)}$ and the process $A=X-M$ satisfies the condition $\sum_{t_{i} \in D_{k}}\left|\Delta A_{t_{i}}\right|^{2} \rightarrow_{P} 0$, and the proof of (i) is finished.

(ii) It is sufficient to use Itô's formula for Dirichlet processes proved by Föllmer (1980) for a function $F(y)=\int_{0}^{y} f(x) \mathrm{d} x$. If $f \in \mathscr{C}^{1}$, then $F \in \mathscr{C}^{2}$ and we have

$$
F\left(X_{t}^{n}\right)=F\left(X_{0}^{n}\right)+\int_{0}^{t} f\left(X_{s}^{n}\right) \mathrm{d} X_{s}^{n}+\frac{1}{2} \int_{0}^{t} f^{\prime}\left(X_{s}^{n}\right) \mathrm{d}\left[X^{n}\right]_{s}
$$

and exactly the same decomposition of $F(X)$. Owing to the continuity of $F, f^{\prime}$,

$$
\left(X^{n}, F\left(X^{n}\right), \int_{0}^{\cdot} f^{\prime}\left(X_{s}^{n}\right) \mathrm{d}\left[X^{n}\right]_{s}\right) \underset{\mathscr{D}}{\rightarrow}\left(X, F(X), \int_{0}^{\cdot} f^{\prime}\left(X_{s}\right) \mathrm{d}[X]_{s}\right),
$$

which completes the proof of (ii). 
(iii) It is evident that the sequences $\left\{X^{n}+Y^{n}\right\}$ and $\left\{X^{n}-Y^{n}\right\}$ also satisfy UTD. On the other hand $\left(X^{n}, Y^{n}, X^{n}+Y^{n}, X^{n}-Y^{n}\right) \rightarrow_{\mathscr{D}}(X, Y, X+Y, X-Y)$ in $\mathbb{C}\left([0, T], \mathbb{R}^{4}\right)$. Finally, by (i),

$$
\left(X^{n}, Y^{n},\left[X^{n}\right],\left[Y^{n}\right],\left[X^{n}+Y^{n}\right],\left[X^{n}-Y^{n}\right]\right) \underset{\mathscr{D}}{\rightarrow}(X, Y,[X],[Y],[X+Y],[X-Y])
$$

in $\mathbb{C}\left([0, T], \mathbb{R}^{6}\right)$ and (iii) follows.

We do not know whether from (i) one can deduce that $X$ is an $\mathscr{F}^{X}$ Dirichlet process. If we want to ensure that $X$ is a Dirichlet process with respect to the given filtration $\mathscr{F}$ it is sufficient to assume that all processes $\left\{X^{n}\right\}$ are adapted to $\mathscr{F}$ and to use the following theorem.

Theorem 2. Assume that $\left\{X^{n}\right\}$ is a sequence of $\mathscr{F}$ Dirichlet processes satisfying UTD (or $\mathrm{UTD}(2))$. If

$$
\sup _{t \leqslant T}\left|X_{t}^{n}-X_{t}\right| \underset{P}{\rightarrow} 0
$$

then $X$ is an $\mathscr{F}$ Dirichlet process (or $X \in \mathscr{D}^{2}$ ) and we have the convergences

$$
\sup _{t \leqslant T}\left|M_{t}^{n}-M_{t}\right| \underset{P}{\rightarrow} 0, \quad \text { and } \quad \sup _{t \leqslant T}\left|A_{t}^{n}-A_{t}\right| \underset{P}{\longrightarrow} 0,
$$

where $M^{n}, M$ and $A^{n}, A$ are martingale and 0-quadratic variation parts of $X^{n}, X$, respectively.

Proof. Let $\left\{N_{m}\right\}$ be a sequence of real numbers such that $N_{m} \uparrow+\infty$ and $\sigma^{n, N_{m}}=\inf \left\{t ;\left|X_{t}^{n}\right| \geqslant N_{m}\right\}=\inf \left\{t ;\left|X_{t}^{n}\right|>N_{m}\right\}, m \in \mathbb{N}$. Then owing to Lemma 1.2 of Stroock and Varadhan (1979) and by the continuous mapping theorem for every $m \in \mathbb{N}, t_{i} \in D_{k}, k \in \mathbb{N}$ we have

$$
\Delta X_{t_{i}}^{n, N_{m}} \underset{P}{\rightarrow} \Delta X_{t_{i}}^{N_{m}} \quad \text { and } \quad \mathrm{E}\left(\Delta X_{t_{i}}^{n, N_{m}} \mid \mathscr{F}_{t_{i-1}}\right) \underset{P}{\rightarrow} \mathrm{E}\left(\Delta X_{t_{i}}^{N_{m}} \mid \mathscr{F}_{t_{i-1}}\right)
$$

Therefore, for $j \geqslant k$,

$$
\begin{aligned}
P\left(\sum_{t_{i} \in D_{k}} \mid\right. & \left.\left.\sum_{u_{l} \in D_{j}^{t_{i-1}, t_{i}}} \mathrm{E}\left(\Delta X_{u_{l}}^{N_{m}} \mid \mathscr{F}_{u_{l-1}}\right)\right|^{2}>\epsilon\right) \\
& \leqslant \liminf _{n \rightarrow+\infty} P\left(\sum_{t_{i} \in D_{k}}\left|\sum_{u_{l} \in D_{j}^{t_{i-1}, t_{i}}} \mathrm{E}\left(\Delta X_{u_{l}}^{n, N_{m}} \mid \mathscr{F}_{u_{l-1}}\right)\right|^{2}>\epsilon\right) \\
& \leqslant \sup _{n} \sup _{j \geqslant k} P\left(\sum_{t_{i} \in D_{k}}\left|\sum_{u_{l} \in D_{j}^{t_{i-1}, t_{i}}} \mathrm{E}\left(\Delta X_{u_{l}}^{n, N_{m}} \mid \mathscr{F}_{u_{l-1}}\right)\right|^{2}>\epsilon\right) \\
& \rightarrow 0, \quad \text { as } k \rightarrow+\infty .
\end{aligned}
$$


Thus, by the arguments of Föllmer (1981), $\left\{\sum_{t_{i} \in D_{k}^{t}} \mathrm{E}\left(\Delta X_{t_{i}}^{N_{m}} \mid \mathscr{F}_{t_{i-1}}\right)\right\}_{k \in \mathbb{N}}$ is a Cauchy sequence for the distance in probability and for every $m \in \mathbb{N}$ there exists a process $A(m)$ such that

$$
\sup _{t \leqslant T}\left|\sum_{t_{i} \in D_{k}^{t}} \mathrm{E}\left(\Delta X_{t_{i}}^{N_{m}} \mid \mathscr{F}_{t_{i-1}}\right)-A(m)_{t}\right| \underset{P}{\rightarrow} 0 .
$$

One can prove that $A(m)_{t}=A(m+1)_{t}$ for $T \leqslant \sigma^{N_{m}}$. Define $A_{t}=A(m+1)_{t}$ for $t \in\left[\sigma^{N_{m}}, \sigma^{N_{m+1}}\left[, m \in \mathbb{N}\right.\right.$ and $M_{t}=X_{t}-A_{t}$. Thus $M_{t}$ is a local martingale and applying (18) we deduce that $\sum_{t_{i} \in D_{k}}\left|\Delta A_{t_{i}}\right|^{2} \rightarrow_{P} 0$. As a consequence, $X$ is a Dirichlet process with respect to $\mathscr{F}$ and the sequence $\left\{X^{n}-X\right\}$ also satisfies UTD. Since $\sup _{t \leqslant T}\left|X_{t}^{n}-X_{t}\right| \rightarrow{ }_{P} 0$, it follows from Theorem 1 (i) that

$$
\left[M^{n}-M\right]_{T}=\left[X^{n}-X\right]_{T} \underset{P}{\rightarrow} 0 .
$$

Hence $\sup _{t \leqslant T}\left|M_{t}^{n}-M_{t}\right| \rightarrow_{P} 0$ and the proof is completed.

\section{Functionals of Dirichlet processes}

In what follows for a given locally integrable function $f_{n}$ we set $F_{n}(y)=\int_{0}^{y} f_{n}(x) \mathrm{d} x, n \in \mathbb{N}$.

Proposition 2. Let $\left\{X^{n}\right\}$ be a tight in $\mathbb{C}([0, T], \mathbb{R})$ sequence of processes satisfying UTD (or UTD (2)). Then, for every sequence $\left\{f_{n}\right\}$ of functions uniformly bounded and equicontinuous on all compact subsets of $\mathbb{R}^{+}$, the sequence $\left\{F_{n}\left(X^{n}\right)\right\}$ is also a sequence of Dirichlet processes, tight in $\mathbb{C}([0, T], \mathbb{R})$ satisfying UTD (or UTD (2)).

Proof. By Proposition 1, $\left\{X^{n}=M^{n}+A^{n}\right\}$ is a sequence of Dirichlet processes such that (3) holds true and the families of random variables $\left\{\left[M^{n}\right]_{T}\right\}$ and $\left\{\sup _{t \leqslant T}\left|A_{T}^{n}\right|\right\}$ are bounded in probability. If we denote

$$
N_{t}^{n}=\int_{0}^{t} f_{n}\left(X_{s}^{n}\right) \mathrm{d} M_{s}^{n} \quad \text { and } \quad B_{t}^{n}=F_{n}\left(X_{t}^{n}\right)-N_{t}^{n}, \quad t \in \mathbb{R}^{+},
$$

then it is clear that $\left\{N^{n}\right\}$ is a sequence of local martingales and the families of random variables $\left\{\left[N^{n}\right]_{q}\right\},\left\{\sup _{t \leqslant q}\left|B_{t}^{n}\right|\right\}$ are bounded in probability. Therefore, owing to Proposition 1, in order to verify that $\left\{F_{n}\left(X^{n}\right)=N^{n}+B^{n}\right\}$ satisfies UTD, it is sufficient to check that

$$
\lim _{k \rightarrow+\infty} \sup _{n} P^{n}\left(\sum_{t_{i} \in D_{k}}\left|\Delta B_{t_{i}}^{n}\right|^{2}>\epsilon\right)=0, \quad \epsilon>0 .
$$

Since $\left\{X^{n}\right\}$ satisfies UTD and the functions $f_{n}$ are uniformly bounded,

$$
\lim _{k \rightarrow+\infty} \sup _{n} P^{n}\left(\sum_{t_{i} \in D_{k}} f_{n}^{2}\left(X_{t}^{n}\right)\left|\Delta A_{t_{i}}^{n}\right|^{2}>\epsilon\right)=0, \quad \epsilon>0 .
$$


On the other hand by the tightness of $\left\{X^{n}\right\}$ and by the equicontinuity of $\left\{f_{n}\right\}$ we have

$$
\lim _{k \rightarrow+\infty} \sup _{n} P^{n}\left(\sum_{t_{i} \in D_{k}}\left|\int_{t_{i}}^{t_{i+1}} f_{n}\left(X_{s}^{n}\right)-f_{n}\left(X_{t_{i}}^{n}\right) \mathrm{d} M_{s}^{n}\right|^{2}>\epsilon\right)=0, \quad \epsilon>0,
$$

and

$$
\begin{aligned}
\lim _{k \rightarrow+\infty} \sup _{n} P^{n} & \left(\sum_{t_{i} \in D_{k}}\left|F_{n}\left(X_{t_{i+1}}^{n}\right)-F_{n}\left(X_{t_{i}}^{n}\right)-f_{n}\left(X_{t_{i}}^{n}\right)\left(X_{t_{i+1}}^{n}-X_{t_{i}}^{n}\right)\right|^{2}>\epsilon\right) \\
& =\lim _{k \rightarrow+\infty} \sup _{n} P^{n}\left(\sum_{t_{i} \in D_{k}}\left|\int_{X_{t_{i}}^{n}}^{X_{t_{i+1}}^{n}} f_{n}(u)-f_{n}\left(X_{t_{i}}^{n}\right) \mathrm{d} u\right|^{2}>\epsilon\right), \\
& \leqslant \lim _{k \rightarrow+\infty} \sup _{n} P^{n}\left(\max _{t_{i} \in D_{k}} \sup _{X_{t_{i}}^{n} \leqslant u<X_{t_{i+1}}^{n}}\left|f_{n}(u)-f_{n}\left(X_{t_{i}}^{n}\right)\right|^{2} \sum_{t_{i} \in D_{k}}\left|\Delta X_{t_{i}}^{n}\right|^{2}>\epsilon\right), \\
& =0, \quad \epsilon>0 .
\end{aligned}
$$

Hence the proof of (19) is completed. By similar arguments we can prove that (4) with $p=2$ implies that the family of random variables

$$
\left\{\sum_{i=1}^{m}\left|B_{s_{i}}^{n}-B_{s_{i-1}}^{n}\right|^{2} ; 0=s_{0} \leqslant \ldots \leqslant s_{m}=T, s_{i} \in D_{k}, m, k, n \in \mathbb{N}\right\}
$$

is bounded in probability and thus the proof is completed.

Corollary 2. Assume that $f$ is a continuous function and $F(y)=\int_{0}^{y} f(x) \mathrm{d} x$. If $X=M+A$ is an $\mathscr{F}$ Dirichlet process (or $X \in \mathscr{D}^{2}$ ), then also $F(X)$ is an $\mathscr{F}$ Dirichlet process (or $F(X) \in \mathscr{D}^{2}$ ) and the local martingale part of $F(X)$ is equal to $\int_{0}^{\cdot} f\left(X_{s}\right) \mathrm{d} M_{s}$.

Proof. Let $\left\{f_{n}\right\}$ be a sequence of functions such that $f_{n} \in \mathscr{C}^{2}$ and

$$
\sup _{|x| \leqslant k}\left|f_{n}(x)-f(x)\right| \rightarrow 0, \quad k \in \mathbb{N} .
$$

Then, owing to Proposition 2, $\left\{F_{n}(X)\right\}$ satisfies UTD (or UTD (2)) and in view of Theorem 2 the result follows.

Corollary 3. If $X, Y$ are $\mathscr{F}$ adapted Dirichlet processes (or $X, Y \in \mathscr{D}^{2}$ ), then $X Y$ is an $\mathscr{F}$ Dirichlet process (or $X Y \in \mathscr{D}^{2}$ ).

Proof. Let $X, Y$ be Dirichlet processes admitting decompositions of the form $X=$ $M+A, Y=N+B$. Clearly $M N$ is an $\mathscr{F}$ semimartingale and, by the inequality

$$
\sum_{t_{i} \in D_{k}}\left|\Delta(A B)_{t_{i}}\right|^{2} \leqslant 2 \sup _{t \leqslant T}\left|A_{t}\right|^{2} \sum_{t_{i} \in D_{k}}\left|\Delta B_{t_{i}}\right|^{2}+2 \sup _{t \leqslant T}\left|B_{t}\right|^{2} \sum_{t_{i} \in D_{k}}\left|\Delta A_{t_{i}}\right|^{2}
$$


$A B$ is an $\mathscr{F}$ adapted process of 0 -quadratic variation; hence it remains to prove that $M B$ and $A N$ are $\mathscr{F}$ Dirichlet processes. To this end, observe that, by applying Corollary 2 with $f(x)=2 x,(M+B)^{2}$ is an $\mathscr{F}$ adapted Dirichlet process. Therefore, $M B=$ $\frac{1}{2}\left\{(M+B)^{2}-M^{2}-B^{2}\right\}$ is also an $\mathscr{F}$ Dirichlet process. Similarly we show that $A N$ is an $\mathscr{F}$ Dirichlet process and the first assertion is proved.

Now, assume that $X, Y \in \mathscr{D}^{2}$. Since for every subdivision $0=s_{0} \leqslant s_{1} \leqslant \ldots \leqslant s_{m}=T$, such that $s_{i} \in D_{k}, m, k \in \mathbb{N}$

$$
\sum_{i=1}^{m}\left|\Delta(X Y)_{s_{i}}\right|^{2} \leqslant \sup _{t \leqslant T}\left|X_{t}\right|^{2} \sum_{i=1}^{m}\left|\Delta Y_{s_{i}}\right|^{2}+2 \sup _{t \leqslant T}\left|Y_{t}\right|^{2} \sum_{i=1}^{m}\left|\Delta X_{s_{i}}\right|^{2},
$$

and $X Y$ is a Dirichlet process it is evident that also $X Y \in \mathscr{D}^{2}$.

\section{Stability of Dirichlet processes of class $\mathscr{D}^{p}, p<2$}

The existence of a stochastic integral for two $\mathscr{F}$ adapted Dirichlet processes, $X, Y$ satisfying (8) and (9) was proved by Bertoin (1989). Below we generalize slightly his results for processes of class $\mathscr{D}^{p}$. As a main tool in the proof we use a stochastic version of some inequality proved by Young (1936).

Let $A_{1}, \ldots, A_{n}, B_{1}, \ldots, B_{n}$ be random variables such that $\mathrm{E}\left|A_{i}\right|^{p}, \mathrm{E}\left|B_{i}\right|^{q}<+\infty$, $i=1, \ldots, n$. Let $S_{p, q}$ be the largest value of the products

$$
\left(\sum_{k=1}^{m} \mathrm{E}\left|\bar{A}_{k}\right|^{p}\right)^{1 / p}\left(\sum_{k=1}^{m} \mathrm{E}\left|\bar{B}_{k}\right|^{q}\right)^{1 / q}
$$

for which $\bar{A}_{k}=A_{i_{k}+1}+\ldots+A_{i_{k+1}} \quad$ and $\quad \bar{B}_{k}=\bar{B}_{i_{k+1}}+\ldots+B_{i_{k+1}}$, where $1=i_{1}<$ $\ldots<i_{k}<\ldots i_{m+1}=m, m \leqslant n$, are the corresponding sums of successive random variables $A_{i}$ and $B_{i}$, respectively.

Lemma 1. Assume that $1 / p+1 / q>1$. Then

(i) there exists an index $k(1 \leqslant k \leqslant n)$, such that

$$
\mathrm{E}\left|A_{k} B_{k}\right| \leqslant\left(\frac{1}{n} \sum_{k=1}^{n} \mathrm{E}\left|A_{k}\right|^{p}\right)^{1 / p}\left(\frac{1}{n} \sum_{k=1}^{n} \mathrm{E}\left|B_{k}\right|^{q}\right)^{1 / q}
$$

(ii)

$$
\mathrm{E}\left|\sum_{1 \leqslant r \leqslant s \leqslant n} A_{r} B_{s}\right| \leqslant\left(1+\zeta\left(\frac{1}{p}+\frac{1}{q}\right)\right\} S_{p, q}
$$

where $\zeta(s)=\sum_{n=1}^{+\infty} n^{-s}, s>1$.

Proof. We follow step by step the proof of the unequalities 2.1 and 5.1 of Young (1936). 
Theorem 3. Let $X, Y$ be two $\mathscr{F}$ adapted Dirichlet processes such that $X \in \mathscr{D}^{2}, Y \in \mathscr{D}^{p}$ for some $p \in[1,2)$. Then there exists an $\mathscr{F}$ Dirichlet processes $\int X_{s} \mathrm{~d} Y_{s} \in \mathscr{D} p$ such that

$$
\sup _{t \leqslant T}\left|\sum_{t_{i} \in D_{k}^{t}} X_{t_{i-1}} \Delta Y_{t_{i}}-\int_{0}^{t} X_{S} \mathrm{~d} Y_{S}\right| \underset{P}{\rightarrow} 0
$$

Proof. Let $X, Y$ be two $\mathscr{F}$ Dirichlet processes admitting decompositions of the form $X=M+A, Y=N+B$. Since the stochastic integrals $\int_{0} X_{s} \mathrm{~d} N_{s}$ and $\int_{0} M_{s} \mathrm{~d} B_{s}=$ $M B-\int_{0}^{\cdot} B_{s} \mathrm{~d} M_{s}$ are well defined, i.e.

$$
\sum_{t_{i} \in D_{k}^{t}} X_{t_{i-1}} \Delta N_{t_{i}} \underset{P}{\rightarrow} \int_{0}^{t} X_{S} \mathrm{~d} N_{s} \quad \text { and } \quad \sum_{t_{i} \in D_{k}^{t}} M_{t_{i-1}} \Delta B_{t_{i}} \underset{P}{\rightarrow} \int_{0}^{t} M_{s} \mathrm{~d} B_{s}
$$

it is sufficient to show that the sequence

$$
\left\{\sum_{t_{i} \in D_{k}^{t}} A_{t_{i-1}} \Delta B_{t_{i}}\right\}_{k \in \mathbb{N}}
$$

is convergent in probability. For simplicity of notation we may and shall assume that $D_{k} \subset D_{k+1}$ and that there exists a constant $K$ for which

$$
\begin{gathered}
\mathrm{E} \sum_{i=1}^{m}\left|\Delta A_{s_{i}}\right|^{2} \leqslant K, \\
\mathrm{E}\left(\sum_{i=1}^{m}\left|\Delta B_{s_{i}}\right|^{p}\right)^{2} \leqslant K,
\end{gathered}
$$

provided that $0=s_{0} \leqslant \ldots \leqslant s_{m}=T, s_{i} \in D_{k}, m, k \in \mathbb{N}$. Then, for $j \geqslant k$,

$$
\begin{aligned}
& \sum_{t_{i} \in D_{k}^{t}} A_{t_{i-1}} \Delta B_{t_{i}}-\sum_{u_{l} \in D_{j}^{t}} A_{u_{l-1}} \Delta B_{u_{l}}=\sum_{t_{i} \in D_{k}^{t}} \sum_{u_{l} \in D_{j}^{t_{i-1}, t_{i}}}\left(A_{t_{i-1}}-A_{u_{l-1}}\right) \Delta B_{u_{l}} \\
& =\sum_{t_{i} \in D_{k}^{t}} \sum_{u_{l} \in D_{j}^{t_{i-1}, t_{i}}} \Delta A_{u_{l}} \Delta B_{u_{l}}-\sum_{t_{i} \in D_{k}^{t}} \sum_{\substack{u_{l} \leqslant u_{m}, u_{l}, u_{m} \in D_{j}^{t_{i-1}, t_{i}}}} \Delta A_{u_{l}} \Delta B_{u_{m}} .
\end{aligned}
$$

Therefore by the Hölder inequality, (21) as well as by Lemma 1 (ii), for $r \in(p, 2)$, 


$$
\begin{aligned}
\operatorname{Esup} & \left|\sum_{t \leqslant T} A_{t_{i} \in D_{k}^{t}} \Delta B_{t_{i}}-\sum_{t_{i} \in D_{j}^{t}} A_{u_{l-1}} \Delta B_{u_{l}}\right| \\
& \leqslant \sup _{t \leqslant T}\left(\sum_{t_{i} \in D_{k}^{t}} \mathrm{E}\left|\sum_{u_{l} \in D_{j}^{t_{i-1}, t_{i}}} \Delta A_{u_{l}} \Delta B_{u_{l}}\right|+\sum_{t_{i} \in D_{k}^{t}} \mathrm{E}\left|\sum_{\substack{u_{l} \leqslant u_{m}, u_{l}, u_{m} \in D_{j}^{t_{i-1}, t_{i}}}} \Delta A_{u_{l}} \Delta B_{u_{m}}\right|\right) \\
& \leqslant K^{1 / 2}\left(\sum_{t_{i} \in D_{k}} \sum_{u_{l} \in D_{j}^{t_{i-1}, t_{i}}} \mathrm{E}\left|\Delta B_{u_{l}}\right|^{2}\right)^{1 / 2}+\sum_{t_{i} \in D_{k}}\left\{1+\zeta\left(\frac{1}{2}+\frac{1}{r}\right)\right\} \bar{S}_{2, r}^{k, i, j},
\end{aligned}
$$

where $\bar{S}_{2, r}^{k, i, j}$ is the largest value of the products

$$
\left(\sum_{n=1}^{m} \mathrm{E}\left|\Delta A_{S_{n}}\right|^{2}\right)^{1 / 2}\left(\sum_{n=1}^{m} \mathrm{E}\left|\Delta B_{S_{n}}\right|^{r}\right)^{1 / r},
$$

for $t_{i-1}=s_{0} \leqslant \ldots \leqslant s_{m}=t_{i}, s_{n} \in \mathscr{D}_{j} m \in \mathbb{N}$. On the other hand by (22) we deduce that

$$
\mathrm{E}\left(\sum_{t_{i} \in D_{k}} \sum_{u_{l} \in D_{j}^{t_{i-1}, t_{i}}}\left|\Delta B_{u_{l}}\right|^{2}\right)^{p} \leqslant \mathrm{E}\left(\sum_{t_{i} \in D_{k}} \sum_{u_{l} \in D_{j}^{t_{i-1}, t_{i}}}\left|\Delta B_{u_{l}}\right|^{p}\right)^{2} \leqslant K
$$

and, for $p^{\prime}=2 p / r>p$,

$$
\mathrm{E}\left(\sum_{t_{i} \in D_{k}} \sum_{n=1}^{m}\left|\Delta B_{s_{n}}\right|^{r}\right)^{p^{\prime}} \leqslant \mathrm{E}\left(\sum_{t_{i} \in D_{k}} \sum_{n=1}^{m}\left|\Delta B_{s_{n}}\right|^{p}\right)^{2} \leqslant K .
$$

Since we can assume that $p>1$, the uniform integrability property for the respective families of random variables follows. Moreover, by continuity of trajectories of $B$,

$$
\lim _{k \rightarrow+\infty} \sup _{j \geqslant k} \sum_{t_{i} \in D_{k}} \sum_{u_{l} \in D_{j}^{t_{i-1}, t_{i}}} \mathrm{E}\left|\Delta B_{u_{l}}\right|^{2} \rightarrow 0
$$

and

$$
\lim _{k \rightarrow+\infty} \sup _{j \geqslant k} \sum_{t_{i} \in D_{k}} \bar{S}_{2, r}^{k, i, j} \rightarrow 0 .
$$

Thus, in particular $\left\{\sum_{t_{i} \in D_{k}^{t}} A_{t_{i-1}} \Delta B_{t_{i}}\right\}_{k \in \mathbb{N}}$ is a Cauchy sequence, and hence convergent in probability. Let $\int_{0}^{t} A_{S} \mathrm{~d} B_{s}$ denote its limit. Note that in fact we have proved that

$$
\sum_{t_{i} \in D_{k}}\left|A_{t_{i-1}} \Delta B_{t_{i}}-\int_{t_{i-1}}^{t_{i}} A_{S} \mathrm{~d} B_{S}\right| \underset{P}{\rightarrow} 0
$$

Let $\pi^{\delta}$ denote a set of subdivisions of $[0, T]$ such that $0=s_{0}^{\delta} \leqslant s_{1}^{\delta} \leqslant \ldots \leqslant s_{m^{\delta}}^{\delta}=T$, $s_{i-1}^{\delta}-s_{i}^{\delta} \leqslant \delta$ where $s_{i}^{\delta} \in D_{k}, m^{\delta}, k \in \mathbb{N}$. The proof of (23) gives more, namely for every $\epsilon>0$ there exists $\delta>0$, with the property 


$$
\sup _{\pi^{\delta}} P\left(\sum_{i=1}^{m^{\delta}}\left|A_{s_{i-1}^{\delta}} \Delta B_{s_{i}^{\delta}}-\int_{s_{i-1}^{\delta}}^{s_{i}^{\delta}} A_{s} \mathrm{~d} B_{S}\right| \geqslant \epsilon\right) \leqslant \epsilon .
$$

Now, let $\pi$ denote a set of all subdivisions of $[0, T]$ such that $0=s_{0} \leqslant s_{1}<\ldots<s_{m}=T$ and $s_{i} \in D_{k}, m, k \in \mathbb{N}$. Since the process $B$ satisfies (7), for every $\epsilon>0$,

$$
\begin{aligned}
& \limsup _{C \rightarrow+\infty} \sup _{\pi} P\left(\sum_{i=1}^{m}\left|\int_{S_{i-1}}^{s_{i}} A_{S} \mathrm{~d} B_{S}\right|^{p} \geqslant 2 C\right) \\
& \leqslant \limsup _{C \rightarrow+\infty} \sup _{\pi} P\left(\sum_{\left\{i ;\left|s_{i}-s_{i-1}\right| \leqslant \delta\right\}}\left|\int_{s_{i-1}}^{s_{i}} A_{S} \mathrm{~d} B_{S}\right|^{p} \geqslant C\right) \\
& +\limsup _{C \rightarrow+\infty} \sup _{\pi} P\left(\sum_{\left\{i ;\left|s_{i} s_{i-1}\right|>\delta\right\}}\left|\int_{s_{i-1}}^{s_{i}} A_{S} \mathrm{~d} B_{S}\right|^{p} \geqslant C\right) \\
& \leqslant \epsilon+\limsup _{C \rightarrow+\infty} \sup _{\pi} P\left(\sum_{t \leqslant T}\left|A_{t}\right| \sum_{i=1}^{m}\left|\Delta B_{s_{i}}\right|^{p} \geqslant 2^{-p} C-\epsilon^{p}\right) \\
& +\limsup _{C \rightarrow+\infty} \sup _{\pi} P\left(\sum_{t \leqslant T}\left|\int_{0}^{t} A_{S} \mathrm{~d} B_{S}\right|^{p} \geqslant 2^{-p}\left(\frac{T}{\delta}\right)^{-1} C\right) \\
& \leqslant \epsilon
\end{aligned}
$$

and the condition (7) for the integral $\int_{0} A_{s} \mathrm{~d} B_{s}$ holds true, too. Moreover, since the local martingale $M$ satisfies (7) with $p=2$, we deduce that $\int_{0}^{\cdot} X_{s} \mathrm{~d} B_{s}=\int_{0} M_{s} \mathrm{~d} B_{s}+\int_{0}^{\cdot} A_{s} \mathrm{~d} B_{s}$. satisfies (7). Finally,

$$
\int_{0} X_{s} \mathrm{~d} Y_{s}=\int_{0} X_{s} \mathrm{~d} N_{s}+\int_{0} X_{s} \mathrm{~d} B_{s}
$$

where $\int_{0}^{\cdot} X_{s} \mathrm{~d} N_{s}$ is a local martingale and $\int_{0}^{\cdot} X_{s} \mathrm{~d} B_{s}$ satisfies (4), and hence $\int_{0}^{\cdot} \mathrm{d} Y_{s} \in \mathscr{D}^{p}$.

Corollary 4. Let $X, Y$ be $\mathscr{F}$ adapted Dirichlet processes such that $X \in \mathscr{D}^{2}, Y \in \mathscr{D}^{p}$, for some $p \in[1,2)$. Then there exists an $\mathscr{F}$ Dirichlet process $\int Y_{s} \mathrm{~d} X_{s} \in \mathscr{D}^{2}$ such that

$$
\sup _{t \leqslant T}\left|\sum_{t_{i} \in D_{k}^{t}} Y_{t_{i-1}} \Delta X_{t_{i}}-\int_{0}^{t} Y_{S} \mathrm{~d} X_{S}\right| \underset{P}{\rightarrow} 0 .
$$

Proof. By Corollary 3, $X Y \in \mathscr{D}^{2}$. Moreover, it follows from Theorem 3 that the process $\int_{0} Y_{s} \mathrm{~d} X_{s}$ defined by the formula

$$
\int_{0}^{t} Y_{s} \mathrm{~d} X_{s}=Y_{t} X_{t}-\int_{0}^{t} X_{s} \mathrm{~d} Y_{s}-[X, Y]_{t}, t \in[0, T],
$$

has the desired properties. 
Corollary 5. Let $K, X, Y$ be $\mathscr{F}$ adapted Dirichlet processes such that $K, X \in \mathscr{D}^{2}, Y \in \mathscr{D}^{p}$, for some $p \in[1,2)$ and let $Z=\int_{0} X_{s} \mathrm{~d} Y_{s}$. Then

$$
\int_{0}^{t} K_{s} \mathrm{~d} Z_{s}=\int_{0}^{t} K_{s} X_{s} \mathrm{~d} Y_{s}, \quad t \in[0, T] .
$$

Proof. By Theorem 3 and Corollary 4 the integrals $\int_{0}^{t} K_{s} \mathrm{~d} Z_{s}$ and $\int_{0}^{t} K_{s} X_{s} \mathrm{~d} Y_{s}$ are well defined as limits in probability of the sums $\left\{\sum_{t_{i} \in D_{k}^{t}} K_{t_{i-1}} \Delta Z_{t_{i}}\right\}$ and $\left\{\sum_{t_{i} \in D_{k}^{t}} K_{t_{i-1}} X_{t_{i-1}} \Delta Y_{t_{i}}\right\}$, respectively. Assume that $Y$ admits the decomposition of the form $Y=N+B$ and let $C=\int_{0} X_{s} \mathrm{~d} B_{s}$. Since the associativity formula for stochastic integrals driven by local martingales is well known, we need to show only that

$$
\int_{0}^{t} K_{s} \mathrm{~d} C_{s}=\int_{0}^{t} K_{s} \mathrm{X}_{s} \mathrm{~d} B_{s}, \quad t \in[0, T] .
$$

By the definition, $\int_{0}^{t} K_{s} \mathrm{~d} C_{s}$ and $\int_{0}^{t} K_{s} X_{s} \mathrm{~d} B_{s}$ are limits of the sums $\left\{\sum_{t_{i} \in D_{k}^{t}} K_{t_{i-1}} \Delta C_{t_{i}}\right\}$ and $\left\{\sum_{t_{i} D_{k}^{t}} K_{t_{i-1}} X_{t_{i-1}} \Delta B_{t_{i}}\right\}$, respectively. On the other hand,

$$
\left|\sum_{t_{i} \in D_{k}^{t}} K_{t_{i-1}} \Delta C_{t_{i}}-\sum_{t_{i} \in D_{k}^{t}} K_{t_{i-1}} X_{t_{i-1}} \Delta B_{t_{i}}\right| \leqslant \sum_{t_{i} \in D_{k}^{t}}\left|K_{t_{i-1}}\right|\left|\int_{t_{i-1}}^{t_{i}} X_{S} \mathrm{~d} B_{s}-X_{t_{i-1}} \Delta B_{t_{i}}\right| ;
$$

hence the results follows by (23).

If we want to make sure that a limit process is a Dirichlet of class $\mathscr{D}^{p}, p \in[1,2)$, it is convenient to consider the following $\operatorname{UTD}(p)$ condition. We say that a sequence of Dirichlet processes $\left\{X^{n}=M^{n}+A^{n}\right\}$ satisfies $\operatorname{UTD}(p)$ condition for $p \in[1,2)$ if

$\operatorname{UTD}(p)$ : the families of random variables $\left\{\left[M^{n}\right]_{T}\right\}$ and

$$
\left\{\sum_{i=1}^{m}\left|A_{s_{i}}^{n}-A_{s_{i-1}}^{n}\right|^{p} ; 0=s_{0} \leqslant \ldots \leqslant s_{m}=T, s_{i} \in D_{k}, m, k, n \in \mathbb{N}\right\}
$$

are bounded in probability.

Remark 2. Since in the case of continuous semimartingales (UT) is satisified if and only if the families of random variables

$$
\left\{\left[M^{n}\right]_{T}\right\},\left\{\operatorname{var}\left(A^{n}\right)_{T}\right\} \text { are bounded in probability }
$$

(see, for example, Kurtz and Protter (1991a) and Mémin and Słomiński (1991)), the condition $\mathrm{UTD}(1)$ is exactly equivalent to UT.

Corollary 6. Assume that $\left\{X^{n}\right\},\left\{Y^{n}\right\}$ are two sequences of Dirichlet processes satisfying the conditions $\mathrm{UTD}(2)$ and $\operatorname{UTD}(p)$ for some $p \in[1,2)$.

(i) If $Y^{n} \rightarrow_{\mathscr{D}} Y$ in $\mathbb{C}([0, T], \mathbb{R})$, then $Y$ is a $\mathscr{G}$ Dirichlet process for some filtration $\mathscr{G}$ such that $\mathscr{F}^{Y} \subset \mathscr{G}, Y \in \mathscr{D}^{p}$ and

$$
\left(Y^{n},\left[Y^{n}\right]\right) \underset{\mathscr{D}}{\rightarrow}(Y,[Y]) \quad \text { in } \mathbb{C}\left([0, T], \mathbb{R}^{2}\right)
$$


(ii) If $\left(X^{n}, Y^{n}\right) \rightarrow_{\mathscr{D}}(X, Y)$ in $\mathbb{C}\left([0, T], \mathbb{R}^{2}\right)$, then

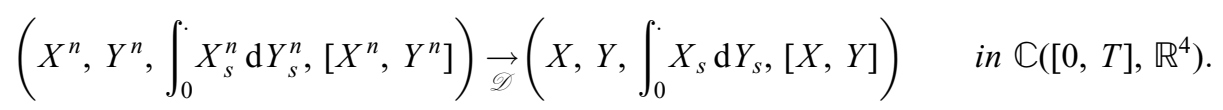

Proof. By using the estimations from the proof of Theorem 3 we observe that, for every $\epsilon>0$

$$
\lim _{k \rightarrow+\infty} \sup _{n} P^{n}\left(\sup _{t \leqslant T}\left|\sum_{t_{i} \in D_{k}^{t}} Y_{t_{i-1}}^{n} \Delta Y_{t_{i}}^{n}-\int_{0}^{t} Y_{s} \mathrm{~d} Y_{s}\right|>\epsilon\right)=0
$$

and

$$
\lim _{k \rightarrow+\infty} \sup _{n} P^{n}\left(\sup _{t \leqslant T}\left|\sum_{t_{i} \in D_{k}^{t}} X_{t_{i-1}}^{n} \Delta Y_{t_{i}}^{n}-\int_{0}^{t} X_{s} \mathrm{~d} Y_{S}\right|>\epsilon\right)=0
$$

In view of (24) and the integration-by-parts formula

$$
\lim _{k \rightarrow+\infty} \sup _{n} P^{n}\left(\left.\sup _{t \leqslant T}\left|\sum_{t_{i} \in D_{k}^{t}}\right| \Delta Y_{t_{i}}^{n}\right|^{2}-[Y]_{t} \mid>\epsilon\right)=0
$$

and the proof of (i) is finished. Finally we deduce (ii) by (i) and (25).

Note that $\operatorname{UTD}(p)$ for $p \in[1,2)$ need not imply UTD. Indeed, (3) need not hold true! However, by the above corollary we deduce that, if $\left\{X^{n}=M^{n}+A^{n}\right\}$ is tight in $\mathbb{C}([0, T], \mathbb{R})$, then $\operatorname{UTD}(p) \Rightarrow \operatorname{UTD}(2)$. Namely, by Corollary 6 (i), $\left\{\left[M^{n}\right]=\left[X^{n}\right]\right\}$ is tight in $\mathbb{C}([0, T], \mathbb{R})$. Hence $\left\{M^{n}\right\}$ and $\left\{A^{n}\right\}$ are tight in $\mathbb{C}([0, T], \mathbb{R})$, too. Therefore in particular

$$
\lim _{k \rightarrow+\infty} \sup _{n} P\left(\max _{t_{i} \in D_{k}}\left|\Delta A_{t_{i}}^{n}\right|>\epsilon\right)=0, \quad \epsilon>0,
$$

and in this case (3) is a consequence of (4).

Corollary 7. Assume that $\left\{X^{n}\right\}$ is a sequence of $\mathscr{F}$ Dirichlet processes satisfying $\operatorname{UTD}(p)$. If

$$
\sup _{t \leqslant T}\left|X_{t}^{n}-X_{t}\right| \underset{P}{\rightarrow} 0
$$

then $X$ is an $\mathscr{F}$ Dirichlet process of class $D^{p}$ and we have the convergences

$$
\sup _{t \leqslant T}\left|M_{t}^{n}-M_{t}\right| \underset{P}{\rightarrow} 0 \quad \text { and } \quad \sup _{t \leqslant T}\left|A_{t}^{n}-A_{t}\right| \underset{P}{\rightarrow} 0
$$

where $M^{n}, M$ and $A^{n}, A$ are martingale and 0 -quadratic variation parts of $X^{n}, X$, respectively.

Proof. This easily follows because of Corollary 6. 


\section{Functionals of semimartingales}

Given $\gamma \in(0,1]$ and $\left\{L_{k}\right\} \subset \mathbb{R}^{+}, \mathbb{L}^{\text {loc }}\left(\gamma ;\left\{L_{k}\right\}\right)$ denotes the class of functions $f$ such that

$$
|f(x)-f(y)| \leqslant L_{k}|x-y|^{\gamma}, \quad|x|,|y| \leqslant k, k \in \mathbb{N} \text {. }
$$

Proposition 3. Let $\left\{X^{n}\right\}$ be a tight-in- $\mathbb{C}([0, T], \mathbb{R})$ sequence of semimartingales satisfying UT. If $\left\{f_{n}\right\}$ is a sequence of functions such that $\left\{f_{n}\right\} \subset \mathbb{L}^{\text {loc }}\left(2 / p-1 ;\left\{L_{k}\right\}\right)$ for some $p \in[1,2),\left\{L_{k}\right\} \subset \mathbb{R}^{+}$and $\left|f_{n}(0)\right| \leqslant C, n \in \mathbb{N}$, for some constant $C>0$, then $\left\{F_{n}\left(X^{n}\right)\right\}$ is a tight-in- $\mathbb{C}([0, T], \mathbb{R})$ sequence of Dirichlet processes satisfying processes satisfying $\operatorname{UTD}(p)$.

Proof. Following the notation from the proof of Proposition 2 and according to the definition of $\operatorname{UTD}(p)$ it is sufficient to prove (20). Without loss of generality we may and shall assume that $\left|f_{n}\right|, \quad \operatorname{var} A_{T}^{n}, \quad\left[M^{n}\right]_{T}, \quad \sup _{t \leqslant T} \mid X_{t}^{n} \leqslant K$ for some constant $K>0$. Fix $0=s_{0} \leqslant$ $\ldots \leqslant s_{m}=T, s_{i} \in D_{k}$, and $m, k, n \in \mathbb{N}$. Then, by the Jensen inequality,

$$
\sum_{i=1}^{m} f_{n}^{p}\left(X_{s_{i}}^{n}\right)\left|\Delta A_{s_{i}}^{n}\right|^{p} \leqslant K^{p}\left(\operatorname{var} A_{T}^{n}\right)^{p} \leqslant K^{2 p} .
$$

Next by the Burkholder-Davis-Gundy and the Hölder inequalities we have

$$
\begin{aligned}
& \sum_{i=1}^{m} \mathrm{E}\left|\int_{s_{i}}^{s_{i+1}} f_{n}\left(X_{s}^{n}\right)-f_{n}\left(X_{s_{i}}^{n}\right) \mathrm{d} M_{s}^{n}\right|^{p} \\
& \quad \leqslant C_{p} \sum_{i=1}^{m} \mathrm{E}\left(\int_{s_{i}}^{s_{i+1}}\left|f_{n}\left(X_{s}^{n}\right)-f_{n}\left(X_{s_{i}}^{n}\right)\right|^{2} \mathrm{~d}\left[M^{n}\right]_{s}\right)^{p / 2} \\
& \quad \leqslant C_{p} \sum_{i=1}^{m} \mathrm{E} \sup _{s_{i} \leqslant s<s_{i+1}}\left|f_{n}\left(X_{s}^{n}\right)-f_{n}\left(X_{s_{i}}^{n}\right)\right|^{p}\left(\left[M^{n}\right]_{s_{i+1}}-\left[M^{n}\right]_{s_{i}}\right)^{p / 2} \\
& \quad \leqslant C_{p}\left(\sum_{i=1}^{m} \mathrm{E}_{s_{i} \leqslant s<s_{i+1}}\left|f_{n}\left(X_{s}^{n}\right)-f_{n}\left(X_{s_{i}}^{n}\right)\right|^{2 p /(2-p)}\right)^{(2-p) / 2}\left(\sum_{i=1}^{m} \mathrm{E}\left(\left[M^{n}\right]_{s_{i+1}}-\left[M^{n}\right]_{s_{i}}\right)\right)^{p / 2} .
\end{aligned}
$$

Since $\left\{f_{n}\right\}$ are Hölder equicontinuous with exponent $2 / p-1$,

$\sum_{i=1}^{m} \mathrm{E}\left(\sup _{s_{i} \leqslant s<s_{i+1}}\left|f_{n}\left(X_{s}^{n}\right)-f_{n}\left(X_{s_{i}}^{n}\right)\right|^{2 p /(2-p)}\right)$ 


$$
\begin{aligned}
& \leqslant \sum_{i=1}^{m} L_{k}^{p} \mathrm{E} \sup _{s_{i} \leqslant s<s_{i+1}}\left|X_{s}^{n}-X_{s_{i}}^{n}\right|^{2} \\
& \leqslant 2 L_{k}^{p} \sum_{i=1}^{m}\left(\mathrm{E} \sup _{s_{i} \leqslant s<s_{i+1}}\left|M_{s}^{n}-M_{s_{i}}^{n}\right|^{2}+\mathrm{E} \sup _{s_{i} \leqslant s<s_{i+1}}\left|A_{s}^{n}-A_{s_{i}}^{n}\right|^{2}\right) \\
& \leqslant 2 L_{k}^{p} \sum_{i=1}^{m}\left\{4 \mathrm{E}\left(\left[M^{n}\right]_{s_{i+1}}-\left[M^{n}\right]_{s_{i}}\right)+\mathrm{E}\left(\operatorname{var} A_{s_{i+1}}^{n}-\operatorname{var} A_{s_{i}}^{n}\right)^{2}\right\} \\
& \leqslant \operatorname{constant}\left(\mathrm{E}\left[M^{n}\right]_{T}+\mathrm{E} C \operatorname{var} A_{T}^{n}\right) .
\end{aligned}
$$

Finally

$$
\begin{aligned}
\sum_{i=1}^{m} \mid F_{n}\left(X_{s_{i+1}}^{n}\right)-F_{n}\left(X_{s_{i}}^{n}\right)-f_{n}\left(X_{s_{i}}^{n}\right) & \left.\left(X_{s_{i+1}}^{n}-X_{s_{i}}^{n}\right)\right|^{p} \\
& =\sum_{i=1}^{m}\left|\int_{X_{s_{i}}^{n}}^{X_{s_{i+1}}^{n}} f_{n}(u)-f_{n}\left(X_{s_{i}}^{n}\right) \mathrm{d} u\right|^{p} \\
& \leqslant \sum_{i=1}^{m}\left|\Delta X_{s_{i}}^{n}\right|^{p-1} \int_{X_{s_{i}}^{n} \wedge X_{s_{i+1}}^{n}}^{X_{s_{i}}^{n} \vee X_{s_{i+1}}^{n}}\left|f_{n}(u)-f_{n}\left(X_{s_{i}}^{n}\right)\right|^{p} \mathrm{~d} u \\
& \leqslant L_{K} \sum_{i=1}^{m}\left|\Delta X_{s_{i}}^{n}\right|^{p-1} \int_{X_{s_{i}}^{n} \wedge X_{s_{i+1}}^{n}}^{X_{s_{i}}^{n} \vee X_{s_{i+1}}^{n}}\left|u-X_{s_{i}}^{n}\right|^{2-p} \mathrm{~d} u \\
& \leqslant L_{K} \sum_{i=1}^{m}\left|\Delta X_{s_{i}}^{n}\right|^{p-1}\left|\Delta X_{s_{i}}^{n}\right|^{2-p}\left|\Delta X_{s_{i}}^{n}\right| \\
& =L_{K} \sum_{i=1}^{m}\left|\Delta X_{s_{i}}^{n}\right|^{2}
\end{aligned}
$$

and the condition UT implies boundedness in probability of the last sum. By using the above (20) easily follows.

Corollary 8. Assume that $f \in \mathbb{L}^{\operatorname{loc}}\left(2 / p-1 ;\left\{L_{k}\right\}\right)$ and set $F(y)=\int_{0}^{y} f(x) \mathrm{d} x$. If $X$ is an $\mathscr{F}$ semimartingale, then $F(X)$ is an $\mathscr{F}$ Dirichlet process of class $\mathscr{D}^{p}$.

Proof. By analogy to the proof of Corollary 2 from Proposition 3 and Theorem 2 the result follows.

If $X$ is a Wiener process, i.e. $X=W$, then it was observed by Wang (1977) and $F(W)$ is a Dirichlet process assuming only that 


$$
\int_{K} f^{2}(x) \mathrm{d} x<+\infty \text { for every compact subset of } \mathbb{R} \text {. }
$$

We shall show that in fact $F(W) \in \mathscr{D}^{2}$. Owing to Theorem 3 and Corollary 2 this fact can be useful for construction of stochastic integral driven by $F(W)$.

Corollary 9. Assume that $f$ satisfies (26) and denote $F(y)=\int_{0}^{y} f(x) \mathrm{d} x$. If $W$ is a Wiener process, then $F(W) \in \mathscr{D}^{2}$.

Proof. By Föllmer et al. (1995), $F(W)_{t}=M_{t}+A_{t}$, where

$$
\begin{aligned}
A_{t}=\frac{1}{2}[f(W), W]_{t} & =\int_{0}^{t} f\left(W_{s}\right) \mathrm{d}^{*} W_{s}-\int_{0}^{t} f\left(W_{s}\right) \mathrm{d} W_{s}, \\
& =A_{t}^{1}-A_{t}^{2}, \quad t \in \mathbb{R}^{+},
\end{aligned}
$$

and $A^{1}=\int_{0}\left(W_{s}\right) \mathrm{d}^{*} W_{s}$ is a backward integral of $f\left(W_{s}\right)$ driven by $W_{s}$. Then it is a simple matter to check that $A^{1}$ and $A^{2}$ satisfy (7) with $p=2$.

\section{Convergence of solutions to stochastic differential equations}

Let $\left\{Z^{n}\right\}$ be a sequence of continuous semimartingales. Consider a sequence of solutions to SDEs of the form

$$
Y_{t}^{n}=\int_{0}^{t} \sigma_{n}\left(Y_{s}^{n}\right) \mathrm{d} Z_{s}^{n}+\int_{0}^{t} \sigma_{n} \sigma_{n}^{\prime}\left(Y_{s}^{n}\right) \mathrm{d}\left[Z^{n}\right]_{s}, \quad t \in \mathbb{R}^{+},
$$

where $\sigma_{n}$ is a function having a continuous derivative $\sigma_{n}^{\prime}, n \in \mathbb{N}$.

Proposition 4. Assume that $\left\{Z^{n}\right\}$ is a sequence of semimartingales satisfying UT and $Z^{n} \rightarrow_{\mathscr{D}} Z$. Let $\left\{Y^{n}\right\}$ be a sequence of solutions to $S D E$ (27), where $\epsilon \leqslant \sigma_{n} \leqslant K$ for some constants $\epsilon, K>0$. If $\sigma_{n} \rightarrow \sigma$ uniformly on compact subsets of $\mathbb{R}$ and $\sigma^{2}$ is a continuous function (or $\sigma^{2} \in \mathbb{L}^{\text {loc }}\left(2 / p-1 ;\left\{L_{k}\right\}\right.$ ) for some $p \in[1,2),\left\{L_{k}\right\} \subset \mathbb{R}^{+}$), then $\left\{Y^{n}\right\}$ is $\mathbb{C}([0, T], \mathbb{R})$ tight and its every limit process $Y$ is an $\mathscr{F}^{Y}$ Dirichlet process of class $\mathscr{D}^{2}$ (or $\left.Y \in \mathscr{D}^{p}\right)$ and satisfies the equation

$$
Y_{t}=\int_{0}^{t} \sigma\left(Y_{s}\right) \mathrm{d} Z_{s}+A_{t}, \quad t \in \mathbb{R}^{+} .
$$

Proof. Define $G_{n}(y)=\int_{0}^{y} \sigma_{n}^{-2}(u) \mathrm{d} u, n \in \mathbb{N}$. Then, for every $n \in \mathbb{N}, X^{n}=G_{n}\left(Y^{n}\right)$ is a solution to the SDE

$$
X_{t}^{n}=\int_{0}^{t} g_{n}\left(X_{s}^{n}\right) \mathrm{d} Z_{s}^{n}, \quad t \in \mathbb{R}^{+},
$$

where $g_{n}(x)=1 / \sigma_{n} \circ G_{n}^{-1}(x)$ because $G_{n}$ is a transformation which allows elimination of 
drift. One can see that $K^{-1} \leqslant g_{n} \leqslant \epsilon^{-1}$ and $g_{n} \rightarrow g$ uniformly on compact subsets of $\mathbb{R}$. Therefore $\left\{X^{n}\right\}$ is $\mathbb{C}([0, T], \mathbb{R})$ tight and satisfies UT. We may and shall assume that

$$
\left(X^{n}, Z^{n}\right) \underset{\mathscr{D}}{\rightarrow}(X, Z), \quad \text { in } \mathbb{C}\left([0, T], \mathbb{R}^{2}\right) .
$$

Then, owing to Theorem 2 from Mémin and Słomiński (1991) for example, $X$ satisfies the equation

$$
X_{t}=\int_{0}^{t} \sigma^{-1}\left(F\left(X_{s}\right)\right) \mathrm{d} Z_{s}, \quad t \in \mathbb{R}^{+},
$$

where $F$ is the inverse function of $G, G(\cdot)=\int_{0}^{\cdot} \sigma^{-2}(u) \mathrm{d} u$. Obviously $X$ is a semimartingale and by Stricker's theorem it is a semimartingale with respect to its natural filtration $\mathscr{F}^{X}$. Let $F_{n}$ denote the inverse function of $G_{n}$. Since $F_{n} \rightarrow F$ uniformly on compact subsets of $\mathbb{R}$, $Y^{n}=F_{n}\left(X^{n}\right) \rightarrow_{\mathscr{D}} F(X)$. On the other hand by a simple calculation it is easy to verify that $F(x)=\int_{0}^{x} f(u) \mathrm{d} u$, where $f(u)=\sigma^{2} \circ F(u)$. Since $f$ is continuous, so, by Corollary 2, $Y=F(X) \in \mathscr{D}^{2}$ with respect to filtration $\mathscr{F}^{Y}=\mathscr{F}^{X}$. Similarly, if $\sigma^{2} \in \mathbb{\mathbb { L }}^{\text {loc }}\left(2 / p-1 ;\left\{L_{k}\right\}\right)$, then, owing to corollary $8, Y \in \mathscr{D}^{p}$. Finally, by (28),

$$
\begin{aligned}
Y_{t}=F\left(X_{t}\right) & =\int_{0}^{t} \sigma^{2}\left(F\left(X_{s}\right)\right) \mathrm{d} X_{s}+A_{t} \\
& =\int_{0}^{t} \sigma\left(Y_{s}\right) \mathrm{d} Z_{s}+A_{t}, \quad t \in[0, T],
\end{aligned}
$$

i.e. $Y$ satisfies $(28)$.

\section{NOTE ADDED IN PROOF}

In the proof of equivalence of UTD and the condition (ii) of Proposition 1 we have used the fact that $D_{k} \subset D_{k+1}$ for $k \in N$. However, in all the subsequent proofs making use of UTD we have used only its characterisation given in Proposition 1. Therefore, if we adopt the condition (ii) of Proposition 1 as the definition of UTD, then all the results of Sections 3-7 remain true irrespective of the fact whether $D_{k} \subset D_{k+1}$ for $k \in N$ or not.

\section{Acknowledgement}

This research was supported by Komitet Badań Naukowych under Grant PB 591/P03/95/08 to L.S.

\section{References}

Aldous, D.J. (1978) Stopping times and tightness. Ann. Probab., 6, 335-340.

Bertoin, J. (1986) Les processus de Dirichlet en tant qu'espace de Banach. Stochastics, 18, 155-168. Bertoin, J. (1989) Sur une intégrale pour les processus à $\alpha$ variation bornée, Ann. Probab., 17, $1521-1535$. 
Föllmer, H. (1980) Calcul d'Itô sans probabilités. Séminaire de Probabilités, pp. 144-150. Lecture Notes Math., 850. Berlin: Springer-Verlag.

Föllmer, H. (1981) Dirichlet processes. Stochastic Integrals, pp. 476-478. Lecture Notes Math., 851. Berlin: Springer-Verlag.

Föllmer, H., Protter, Ph. and Shiryaev, A.N. (1995) Quadratic covariation and an extension of Itô's formula. Bernoulli, 1, 149-169.

Jacod, J. (1980) Convergence en loi de semimartingales et variation quadratique. Séminaire de Probabilités, pp. 547-560. Lecture Notes Math., 850. Berlin: Springer-Verlag.

Jacod, J. and Shiryayev, A.N., (1987) Limit Theorems for Stochastic Processes. Berlin: Springer-Verlag.

Jakubowski, A., Mémin, J. and Pagès, G. (1989) Convergence en loi des suites d'intégrales stochastiques sur l'espace $D^{1}$ de Skorokhod. Probab. Theory Related Fields, 81, 111-137.

Kurtz, T.G. and Protter, P. (1991a) Weak limit theorems for stochastic integrals and stochastic differential equations, Ann. Probab., 19, 1035-1070.

Kurtz, T.G. and Protter, P. (1991b) Wong-Zakai corrections, random evolutions and simulation schemes for SDE's. Proceedings of the Conference in Honor of Moshe Zakai's 65th Birthday, Stochastic Analysis, Haifa, 1991, pp. 331-346. Boston: Academic Press.

Mémin, J. and Słomiński, L. (1991) Condition UT et stabilité en loi des solutions d'équations différentielles stochastiques. Séminaire de Probabilités XXV, pp. 162-177. Lecture Notes Math. 1485. Berlin: Springer-Verlag.

Rozkosz, A. and Słomiński, L. (1998) Extended convergence and Dirichlet processes. Stochastics Stochastics Rep., 65, 79-109.

Słomiński, L. (1989) Stability of strong solutions of stochastic differential equations. Stochastic Processes Applic., 31, 173-202.

Słomiński, L. (1996) Stability of stochastic differential equations driven by general semimartingales, Dissert. Math., 349, 1-113.

Stricker, C. (1985) Loi de semimartingales et critères de compacité. Séminaire de Probabilités XIX, pp. 209-218. Lecture Notes Math. 1123. Berlin: Springer-Verlag.

Stroock, D.W. and Varadhan, S.R.S. (1979) Multidimensional Diffussion Processes. New York: Springer-Verlag.

Young, L.C. (1936) An inequality of the Hölder type, connected with Stieltjes integration, Acta Math., 67, 251-282.

Wang, A.T. (1977) Quadratic variation of functionals of Brownian motion. Ann. Probab., 5, 756-769.

Received January 1997 and revised September 1997 